\title{
Osteologia craniana de Coraciiformes (Aves)
}

\author{
Márcia C. Pascotto ${ }^{\text {; }}$ Elizabeth Höfling ${ }^{2}$ \& Reginaldo J. Donatelli ${ }^{3}$
}

\author{
1 Departamento de Ciências Biológicas e da Saúde, Instituto de Ciências e Letras do Médio Araguaia, Universidade Federal de \\ Mato Grosso. Rodovia MT-100, Km 3,5, 78696-000 Pontal do Araguaia, Mato Grosso, Brasil. Email mcpascot@ufmt.com \\ 2 Departamento de Zoologia, Instituto de Biociências, Universidade de São Paulo. Rua do Matão, Travessa 14, 101, \\ 05508-900 São Paulo, Brasil. E-mail ehofling@ib.usp.br \\ ${ }^{3}$ Laboratório de Vertebrados, Departamento de Ciências Biológicas, Faculdade de Ciências, Universidade Estadual Paulista. \\ Caixa Postal 473, 17001-970 Bauru, São Paulo, Brasil. E-mail rjdonat@yahoo.com.br
}

\begin{abstract}
Cranial osteology of Coraciiformes (Aves). The families Alcedinidae (kingfishers), Momotidae (motmots), Todidae (todies), Meropidae (bee-eaters), Coraciidae (rollers), Brachypteraciidae (ground-rollers), Leptosomidae (cuckoo-rollers), Phoeniculidae (woodhoopoes), Upupidae (hoopoes) and Bucerotidae (hornbills) are traditionally grouped in the Order Coraciiformes, but no external morphological character is commom to the whole order. A comparative study of the cranial osteology of the Coraciiformes seeks to find characters capable to diagnose the order or groups of taxa, serving yet as a source to provide characters for future phylogenetic analysis. As established by the external morphological data, the cranial osteology has ratified the broad morphological divergence among the Coraciiformes taxa. Only two characters are common to the Order as a whole, as the presence of the laterosphenoid fossa and the absence of the suprameatic process, but these characters are not exclusive of the order. Primary homologies were found, showing similarities among several families, such as: the craniofacial flexion zone is an indefinite region in adults of the Coraciidae, Leptosomidae, Phoeniculidae, Upupidae and Bucerotidae; the temporal fossa has intermediate development and deepy in the Momotidae, Meropidae, Coraciidae, Brachypteraciidae and Bucerotidae; the lacrimal bone is absent in the Momotidae, fused with the ectethmoid in adults of the Upupidae, Phoeniculidae and Bucerotidae, and present and free in the Alcedinidae, Todidae, Meropidae, Coraciidae, Brachypteraciidae and Leptosomidae; and the 0 retroarticular process of the mandible is developed in the Upupidae, Phoeniculidae and Bucerotidae. KEY WORDS. Mandible; morphology; skull.
\end{abstract}

RESUMO. Dez famílias são tradicionalmente agrupadas na Ordem Coraciiformes, Alcedinidae (martins-pescadores), Momotidae (udus e juruvas), Todidae ("todies"), Meropidae (abelharucos), Coraciidae (rolieiros), Brachypteraciidae ("ground-rollers"), Leptosomidae ("cuckoo-rollers"), Phoeniculidae ("woodhoopoes"), Upupidae (poupas-comuns) e Bucerotidae (calaus), mas não há caracteres na morfologia externa que sejam comuns a todos os membros da ordem. Assim, este trabalho apresenta uma comparação da osteologia craniana das espécies de Coraciiformes com a finalidade de encontrar caracteres osteológicos que possam diagnosticar a ordem ou grupos de táxons de Coraciiformes, servindo ainda como uma fonte de dados para futuras análises filogenéticas. Como constatado por dados da morfologia externa, os caracteres da osteologia craniana ratificaram a diversidade morfológica existente entre os táxons da Ordem Coraciiformes, sendo difícil diagnosticá-la ou encontrar caracteres comuns a todos os seus membros. Apenas dois caracteres são comuns à ordem, tais como a presença da fossa lateroesfenóide e a ausência do processo suprameático, embora tais caracteres também sejam encontrados em outros grupos de aves. Homologias primárias foram encontradas, indicando similaridades entre diversas famílias, tais como: a zona flexória craniofacial é oclusa nos indiviíduos adultos de Coraciidae, Leptosomidae, Phoeniculidae, Upupidae e Bucerotidae; a fossa temporal tem desenvolvimento e profundidade intermediários em Momotidae, Meropidae, Coraciidae, Brachypteraciidae e Bucerotidae; o lacrimal está ausente em Momotidae, fundido com o ectetmóide nos adultos de Upupidae, Phoeniculidae e Bucerotidae, e presente e distinto em Alcedinidae, Todidae, Meropidae, Coraciidae, Brachypteraciidae e Leptosomidae; e o processo retroarticular da mandíbula é desenvolvido em Upupidae, Phoeniculidae e Bucerotidae.

PALAVRAS-CHAVE. Crânio, morfologia, mandíbula. 
Apesar de muitos autores diagnosticarem a ordem Coraciiformes com base principalmente na morfologia externa ou em dados comportamentais, torna-se difícil reconhecer um membro da ordem baseado simplesmente em caracteres da morfologia externa (como por exemplo, na coloração da plumagem, no tamanho corpóreo, na forma e no tamanho do bico) em função da ampla diversidade morfológica do grupo, além da incerteza sobre o monofiletismo da ordem Coraciiformes (sensu WetMore 1960). Morfologicamente, os Coraciiformes são caracterizados como aves de plumagem colorida e brilhante (Forshaw 1998), principalmente em tons de verde e azul, ou sendo aves com cabeça grande, pescoço e pernas curtas e a maioria com bico longo e largo (FRY \& FrY 1999). A maioria das espécies de Coraciiformes não possui dimorfismo sexual na coloração da plumagem e exibe sindactilia (Fry \& Fry 1999), na qual os dedos 2, 3 e 4 são interligados na base pela membrana interdigital, embora haja modificações em algumas famílias. O palato dos Coraciiformes é desmognato (exceto em Todidae), caracterizado basicamente pela completa fusão mediana dos maxilopalatinos, diretamente ou pela intermediação do septo nasal, enquanto que o vômer é ausente ou rudimentar ou, quando presente, extremamente delgado e com a extremidade distal afilada.

Tradicionalmente, a ordem Coraciiformes reúne dez famílias, segundo Wetmore (1960): Alcedinidae (martins-pescadores), Momotidae (udus e juruvas), Todidae ("todies"), Meropidae (abelharucos), Coraciidae (rolieiros), Brachypteraciidae ("ground-rollers"), Leptosomidae ("cuckoorollers"), Phoeniculidae ("woodhoopoes"), Upupidae (poupascomuns) e Bucerotidae (calaus).

Os martins-pescadores (Alcedinidae) estão divididos em três subfamílias, Alcedininae, Cerylinae e Daceloninae, e apresentam distribuição mundial, não sendo encontrados apenas em altas latitudes e ilhas isoladas (Fry \& Fry 1999). Das 92 espécies (Woodall 2001), apenas seis espécies de Cerylinae, pertencentes a dois gêneros (Chloroceryle Kaup, 1848 e Megaceryle Kaup, 1848), ocorrem no Novo Mundo, sendo as demais 86 espécies, três de Cerylinae, 22 de Alcedininae e 61 de Daceloninae, distribuídas pelo Velho Mundo. As dez espécies de udus e juruvas (Momotidae), reunidas em seis gêneros, são aves exclusivamente neotropicais (SNOw 2001), enquanto que as cinco espécies de Todidae, reunidas em um único gênero Todus Brisson, 1760, estão restritas às ilhas do Caribe (Forshaw 1998, Kepler 2001). Os abelharucos (Meropidae) reúnem cerca de 25 espécies em três gêneros e estão restritos ao Velho Mundo, sendo a região Afrotropical aquela com maior abundância e riqueza específica (ForshaW 1998, Fry 2001a, Fry \& Fry 1999). Os rolieiros (Coraciidae) reúnem 12 espécies em dois gêneros, sendo que a maioria das espécies distribui-se pelas regiões tropicais do Velho Mundo (Fry 2001b). Já a família Brachypteraciidae agrupa cinco espécies em três gêneros e é endêmica de Madagascar (LANGRAND 2001). Leptosomidae e Upupidae são duas famílias monotípicas, unicamente para recepção de Leptosomus discolor (Hermann, 1783) e Upupa epops Linnaeus, 1758 , respectivamente, sendo que $L$. discolor ocorre em Madagascar e nas Ilhas Comores (ForsHaw 1998, MoRRIS \& Hawkins 1998, Goodman 2001), enquanto que U. epops distribui-se amplamente pela África, Eurásia e pelo sudeste da Ásia (Forshaw 1998, Kristin 2001). As oito espécies de Phoeniculidae, pertencentes a dois gêneros, são endêmicas da região sul do Deserto do Saara, na África (Forshaw 1998, Ligon 2001). Os calaus (Bucerotidae) estão representados por 54 espécies agrupadas em duas subfamílias, de acordo com seu modo de vida: Bucorvinae, recepcionando apenas duas espécies do gênero Bucorvus Lesson, 1830 \{B. cafer (Schlegel, 1862) [ = B. leadbeateri (Vigors, 1825)] e B. abyssinicus (Boddaert, 1783)\} devido ao seu modo de vida basicamente terrícola; e Bucerotinae (52 espécies em 13 gêneros), as quais estão aptas a viverem em ambientes florestais (KEMP 2001). Os calaus são encontrados desde a região sub-Saara da África até a Índia e ilhas do arquipélago da Malásia, como também em Papua Nova Guiné e na Ilha Salomão (Kemp 1995).

Dados morfológicos, especialmente baseados no esqueleto, foram muito usados desde o século XIX para se estabelecer relações ou semelhanças entre os inúmeros táxons de Aves, muitas vezes por razões puramente práticas, seja pela grande representatividade em coleções osteológicas ou pela facilidade da observação dos dados. Dentre as aves, o crânio é a unidade esquelética que mais apresenta variações morfológicas entre as famílias e ordens, refletindo especializações aos mais variados hábitos alimentares - embora muitas particularidades estejam restritas à ranfoteca.

Além de existir poucos estudos sobre a osteologia craniana dos Coraciiformes, nenhum deles é muito abrangente: Lowe (1948) descreveu uma extensa lista de caracteres anatômicos com a finalidade de diagnosticar a ordem Coraciiformes, incluindo apenas alguns caracteres osteológicos cranianos, mas seu estudo foi baseado principalmente em Coracias garrulus (Linnaeus, 1758), espécie-tipo da ordem usada como referencial para comparação em outros estudos. Cracraft (1968) realizou um importante estudo sobre o complexo lacrimal-ectetmóide das Aves, descrevendo os padrões encontrados para as famílias de Coraciiformes. Posteriormente, CrACRAFT (1971) realizou uma excelente descrição ostelógica tanto craniana quanto póscraniana, mas apenas para as famílias Coraciidae, Brachypteraciidae e Leptosomidae. Já Feduccia (1975a, b) descreveu os tipos de "columella auris" encontrados nas Aves, enquanto que Burton (1978) preocupou-se em descrever as variações da emarginação do basiesfenóide ("basisphenoid notch") nos martins-pescadores (Alcedinidae). BURTON (1984) realizou uma importante contribuição para o conhecimento da anatomia craniana dos Coraciiformes e Piciformes, mas os detalhes osteológicos e as variações interespecíficas ainda são pouco representativos. Flausino et al. (1999) realizaram um estudo detalhado da osteologia e miologia cranianas de Momotus momota (Linnaeus, 1766) (Momotidae), Pascotto \& Donatelli (2003) descreveram comparativamente a osteologia craniana de todas as espécies de Momotidae e Pascotto et al. (2006) procuraram diferenciar Megaceryle torquata de Ceryle rudis com base na 
osteologia. Outros dados gerais sobre a anatomia dos Coraciiformes foram mencionados em trabalhos anatômicos mais amplos, como nos trabalhos clássicos de STRICKLANd (1843), Sclater (1865), Murie (1872), Garrod $(1875,1878)$, Forbes $(1880,1882)$, SeEbohm (1890), Beddard (1896) e Verheyen (1955).

Segundo Zusi (1981) e Livezey \& Zusi (2001), a diversidade morfológica é uma excelente fonte de caracteres utilizada para a construção de árvores filogenéticas, mas o crânio das aves poderia ser uma fonte ainda mais segura na análise cladística se novos caracteres e evidências anatômicas de homologias fossem encontradas. Para tanto, o autor enfatiza a necessidade de mais dados detalhados sobre as estruturas do crânio e seus órgãos associados. Assim, além da utilização da osteologia craniana como uma fonte de caracteres para a análise filogenética da ordem Coraciiformes, este estudo procurou responder a duas questões principais: 1) Seria possível diagnosticar a ordem e as famílias de Coraciiformes por meio da osteologia craniana? 2) Há variações genéricas ou específicas na osteologia craniana de Coraciiformes? Com base nessas premissas centrais, procurou-se comparar os resultados obtidos com outros estudos encontrados na literatura, tanto para Coraciiformes quanto para outros grupos de Aves.

\section{MATERIAL E MÉTODOS}

Para a descrição e a comparação da osteologia craniana foram utilizados um total de 490 espécimes pertencentes a 149 espécies e 41 gêneros das famílias tradicionalmente agrupadas na ordem Coraciiformes (Apêndice), segundo a nomenclatura taxonômica de Sibley \& Monroe (1990). A classificação utilizada seguiu a proposta de WeTMORE (1960), a qual considera as tradicionais famílias de Coraciiformes: Alcedinidae (Alcedininae, Cerylinae e Daceloninae), Todidae, Momotidae, Meropidae, Coraciidae, Brachypteraciidae, Leptosomidae (= Leptosomatidae), Upupidae, Phoeniculidae e Bucerotidae.

A metodologia adotada para o estudo da osteologia craniana segue aquela descrita por Pascotto \& Donatelli (2003). Para medidas e comparações de determinadas estruturas cranianas, foi utilizado um paquímetro digital Mitutoyo 500-143B, com capacidade de resolução de $0,01 \mathrm{~mm}$ a $150 \mathrm{~mm}$. Machos e fêmeas foram tratados indiscriminadamente, uma vez que não foi encontrado dimorfismo sexual em relação ao tamanho do crânio e de estruturas, salvo raras exceções. Quando observado dimorfismo sexual para determinada estrutura, machos e fêmeas foram considerados amostragens distintas. As imagens dos crânios foram reproduzidas por meio de uma câmera fotográfica digital Pentax Optio MX (zoom óptico e digital 10x, $3,2 \mathrm{MP})$ para ilustração posterior.

A nomenclatura osteológica seguiu basicamente a Nomina Anatomica Avium (BAumel \& Witmer 1993), com modificações. O termo "processo zigomático" adotado por BAUMEL \& WitMER (1993) e utilizado por Pascotto \& Donatelli (2003) foi aqui substituído pelo termo processo esquamosal, como sugerido por JolLIE (1957) e seguido por outros autores (HöfLING \& GASC 1984,
Donatelli 1992, 1996, Posso \& Donatelli 2006), pelo fato do termo zigomático estar associado ao arco zigomático dos mamíferos. O termo "lobos parietais", também sugerido por BAUMEL \& Witmer (1993) e utilizado por Pascotto \& Donatelli (2003), segue a proposição de Posso \& Donatelli (2006) que, por meio da análise de espécimes de cucos (Cuculiformes) jovens, diagnosticou a participação do osso frontal na formação dos lobos parietais devendo a região, portanto, ser denominada de "prominentia frontoparietalis".

Como o lacrimal pode estar extremamente reduzido e fundido com o frontal ou ainda amplamente fundido com o ectetmóide, torna-se difícil determinar, sem um estudo embriológico ou de espécimes jovens, tanto o lacrimal como o ectetmóide em alguns grupos de Coraciiformes, como nos Momotidae, Upupidae e Phoeniculidae. Assim, convencionouse neste trabalho a utilização do termo "típico lacrimal" para os grupos nos quais é possível visualizar e identificar os processos supra-orbital e orbital do lacrimal. Em Bucerotidae foi possível confirmar a completa fusão dos ossos lacrimal e ectetmóide por meio do estudo de indivíduos jovens.

A terminologia fontículo interorbital foi considerada apenas quando a abertura no septo interorbital mostrava-se reduzida ou em forma de um forame; quando tal abertura apresentava-se desenvolvida ou extremamente desenvolvida, ocupando mais da metade ou quase todo o septo interorbital, foi empregado o termo fenestra interorbital, proposto por Zusi (1978) e também adotado por JAMES (2004).

\section{RESULTADOS}

\section{Ossa Cranii}

A região frontal é levemente côncava em Alcedininae e Todidae (F: Fig. 1), plana em Bucerotidae (F: Fig. 2) e côncava nos demais grupos, ocorrendo variações quanto ao grau de desenvolvimento em Daceloninae, Momotidae e Meropidae.

A zona flexória craniofacial dos Coraciiformes apresenta-se completa, exceto em Coraciidae (ZFC: Fig. 3), Leptosomidae, Phoeniculidae, Upupidae e Bucerotidae, nos quais há tendência à oclusão nos indivíduos mais velhos. Já o maior desenvolvimento da zona flexória craniofacial é encontrada nos martins-pescadores da subfamília Cerylinae (ZFC: Fig. 4), a qual se mostra ampla rostrocaudalmente, possuindo a maior largura, dentre todos os Coraciiformes, mas com algumas variações. Em Megaceryle torquata (Linnaeus, 1766) (ro: Fig. 4) e no espécime USNM 318238 de M. lugubris (Temminck, 1834) há uma região ossificada entre a zona flexória craniofacial e a sutura frontonasolacrimal, enquanto que no espécime USNM 560529 de M. maxima (Pallas, 1769) a zona flexória craniofacial é inconspícua lateralmente, sendo evidente apenas na região mediana. Em Ceryle rudis (Linnaeus, 1758) a zona flexória craniofacial é completa, mas sua maior amplitude é observada na região mediana. Em M. alcyon (Linnaeus, 1758) há variação individual em sua amplitude, sendo que apenas o espécime AMNH 24596 assemelha-se ao padrão encontrado em C. rudis. 
A região da Prominentia frontoparietalis é desenvolvida em Upupidae e Phoeniculidae (PF: Fig. 5) e com desenvolvimento menor nos demais grupos. A depressão orbital praticamente inexiste, com exceção de Momotidae (Pascotto \& Donatelli 2003: figs 4, 5, 8), Coraciidae, Leptosomidae e Brachypteraciidae.

A maior largura da caixa craniana, na região do processo pós-orbital, excede cerca de 2,5 vezes a menor largura da região frontal (= região orbital) em Leptosomidae (Fig. 6) e Phoeniculidae, e cerca de 1,8 vez em Upupidae. Nos Bucerotidae (Fig. 2) a caixa craniana tem aproximadamente a mesma amplitude que a região orbital. Dentre os martins-pescadores da subfamília Alcedininae, a caixa craniana excede cerca de três vezes em Alcedo websteri (Hartert, 1898), cerca de 3,5 vezes em A. atthis (Linnaeus, 1758) (Fig. 7), A. cristata Pallas, 1764, A. semitorquata Swainson, 1823, A. azurea Latham, 1801, A. quadribrachys Bonaparte, 1850, A. meninting Horsfield, 1821, Ceyx erithacus (Linnaeus, 1758), C. rufidorsa Strickland, 1847 e C. fallax (Schlegel, 1866); cerca de quatro vezes em C. melanurus (Kaup, 1848), Ispidina picta (Boddaert, 1783) e cerca de 4,5 vezes em A. leucogaster (Fraser, 1843), A. cyanopecta Lafresnaye, 1840, C. lepidus Temminck, 1836 e I. lecontei Cassin, 1856. Já nos Cerylinae, ela expande cerca de 3,3 vezes em Chloroceryle americana (Gmelin, 1788) e C. aenea (Pallas, 1764), e cerca de 2,5 vezes nas demais espécies, enquanto que nos martins-pescadores da subfamília Daceloninae ocorre variação apenas em Todirhamphus winchelli (Sharpe, 1877), em que a caixa craniana excede cerca de 4,3 vezes a menor largura da região frontal; nas demais espécies ela expande cerca de 2,5 a três vezes. Em Meropidae, a caixa craniana é cerca de 2,5 vezes mais larga que a região frontal em Merops bullockoides Smith, 1834, M. pusillus Müller, 1776, M. muelleri (Cassin, 1857), M. variegatus Vieillot, 1817 (Fig. 8), M. hirundinaceus Lichtenstein, 1793, M. oreobates (Sharpe, 1892), M. orientalis Latham, 1801, M. albicollis Vieillot, 1817, M. ornatus Latham, 1801 e M. philippinus Linnaeus, 1766; e cerca de duas vezes em Nyctyornis amictus (Temminck, 1824) (Fig. 9), N. athertoni (Jardine \& Selby, 1830), M. malimbicus Shaw, 1806, M. bulocki Vieillot, 1817, M. nubicus Gmelin, 1788, M. superciliosus Linnaeus, 1766, M. apiaster Linnaeus, 1758 e M. gularis Shaw, 1798. Não comparável com Meropogon forsteni Bonaparte, 1850 em virtude da região orbital estar danificada. Dentre os Coraciiformes, o maior estreitamento da região orbital é observado em Todidae, sendo que a caixa craniana excede cerca de sete vezes a largura da região frontal em Todus todus (Linnaeus, 1758), cerca de 6,5 vezes em T. multicolor Gould, 1837, T. angustirostris Lafresnaye, 1851 e T. mexicanus Lesson, 1838, e cerca de 5,5 vezes em T. subulatus Gray, 1847 (Fig. 1). Nos Coraciidae (Fig. 3) ela é cerca de 2,5 vezes mais ampla que a região frontal, exceto em Eurystomus azureus Gray, 1860 em que excede cerca de 1,7 vez, enquanto que em Brachypteraciidae a maior largura da caixa craniana é encontrada em Brachypteracias leptosomus (Lesson, 1833) (Fig. 10) e Atelornis crossleyi Sharpe, 1875, em que ela é cerca de 3,7 vezes mais larga que a região frontal, enquanto que em $A$. pittoides
(Lafresnaye, 1834) ela excede cerca de 2,8 vezes. Para as variações de Momotidae, consulte Pascotto \& Donatelli (2003).

A fossa temporal apresenta-se rasa e ampla em Phoeniculidae (FT: Fig. 5), Todidae (FT: Fig. 11), Leptosomidae e Upupidae, sendo nestes grupos praticamente plana. Ela é rasa, porém com a porção lateral mais profunda que a porção medial em Momotidae (Pascotto \& Donatelli 2003: figs 4-10), Meropidae (FT: Figs 8, 9), Coraciidae (FT: Fig. 12), Brachypteraciidae (FT: Figs 10, 13) e na maioria das espécies de Bucerotidae, exceto em Buceros vigil (Forster, 1781) em que ela tem o maior desenvolvimento e a maior profundidade, sendo ainda ampla e profunda, especialmente na região lateral, e longa no sentido lateromedial, existindo uma pequena área plana entre as fossas temporais direita e esquerda. Em Cerylinae (FT: Figs 4, 14), Alcedininae (FT: Fig. 7) e Daceloninae (FT: Fig. 24) a fossa temporal tem a maior expansão dorsal, sendo ainda mais longa que larga e com a maior profundidade por toda a sua extensão, com alguma variação específica. A fossa subtemporal é reduzida, mas o menor desenvolvimento é encontrado em duas espécies de martins-pescadores da subfamília Daceloninae [Pelargopsis capensis (Linnaeus, 1766) e P. melanorhyncha (Temminck, 1826)], em que a base ventral é mais estreita. Em Leptosomidae a fossa subtemporal mostra-se inconspícua, não sendo possível distinguir seus limites em função das cristas que a delimitam serem inconspícuas. O processo suprameático está ausente nos Coraciiformes.

O processo esquamosal mostra-se extremamente reduzido, praticamente sem projeção ventral em Leptosomidae e Phoeniculidae (pS: Fig. 5); curto, com cerca de $30 \%$ ou $1 / 3$ da distância desde sua base no esquamosal até o arco jugal em Coraciidae (pS: Fig. 12), Alcedininae (pS: Fig. 15), Cerylinae (os: Fig. 16), Daceloninae (pS: Fig. 17), Meropidae (pS: Fig. 18) e Upupidae; e com desenvolvimento maior, com cerca de metade da distância desde o esquamosal até o arco jugal em Todidae (pS: Fig. 11), Momotidae e Brachypteraciidae (pS: Figs 13, 19). Em Bucerotidae o processo esquamosal é espesso, com a base dorsal ampla e o ápice distal afilado em todas as espécies, mas ocorre variação com relação ao seu desenvolvimento. Ele tem cerca de $70 \%$ de desenvolvimento em Buceros vigil, cerca de $60 \%$ em Anorrhinus galeritus (Temminck, 1831) e Buceros hydrocorax Linnaeus, 1766, e com desenvolvimento que pode variar entre $35 \%$ e $50 \%$ nas demais espécies. Já em Buceros bicornis Linnaeus, 1758 ocorre variação individual, sendo que no espécime macho apresenta-se mais desenvolvido, com cerca de $50 \%$ de desenvolvimento desde a base no esquamosal até o arco jugal, contra cerca de $25 \%$ no espécime fêmea.

O processo pós-orbital é extremamente reduzido em Phoeniculidae (pop: Fig. 5) e Upupidae, praticamente sem projeção; curto, com cerca de $25 \%$ ou menos da distância desde sua base na caixa craniana até o arco jugal em Alcedininae (pop: Fig. 15), Daceloninae (pop: Fig. 17), Cerylinae (pop: Fig. 16), Todidae (pop: Fig. 11) e Meropidae (pop: Fig. 18). Em Brachypteraciidae (pop: Figs 13, 19) mostra-se desenvolvido, ocupando cerca de $90 \%$ desde sua base no esquamosal até o arco jugal. Já 

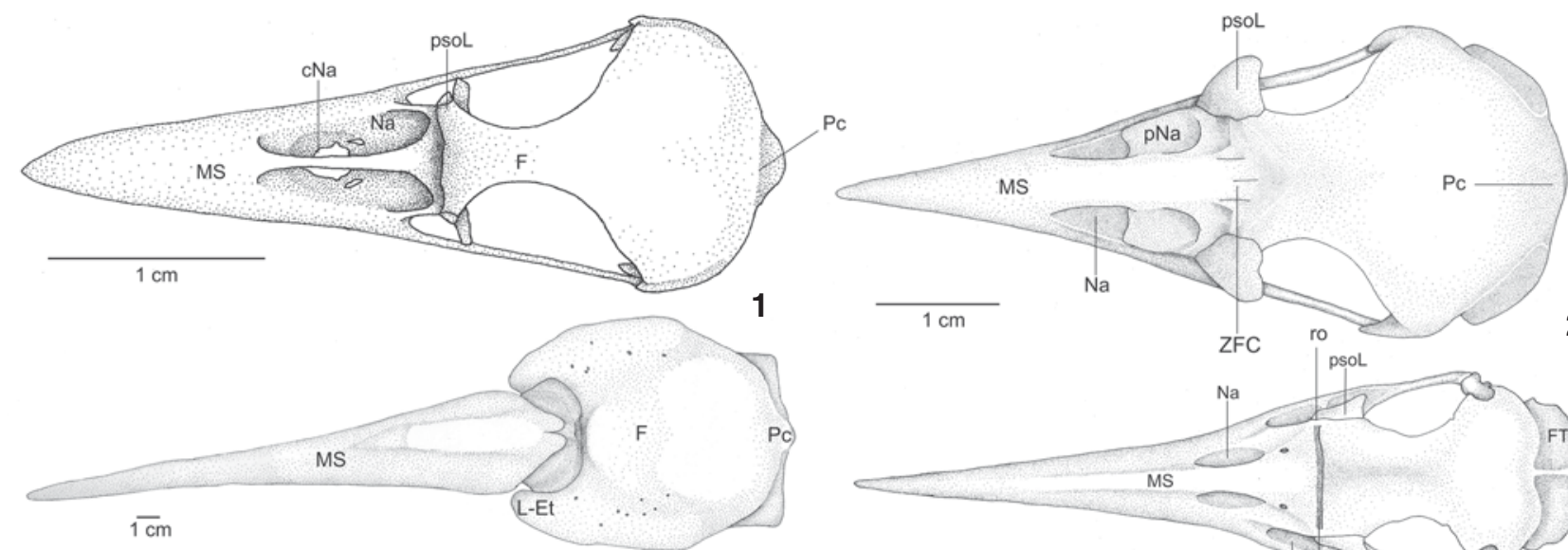

3
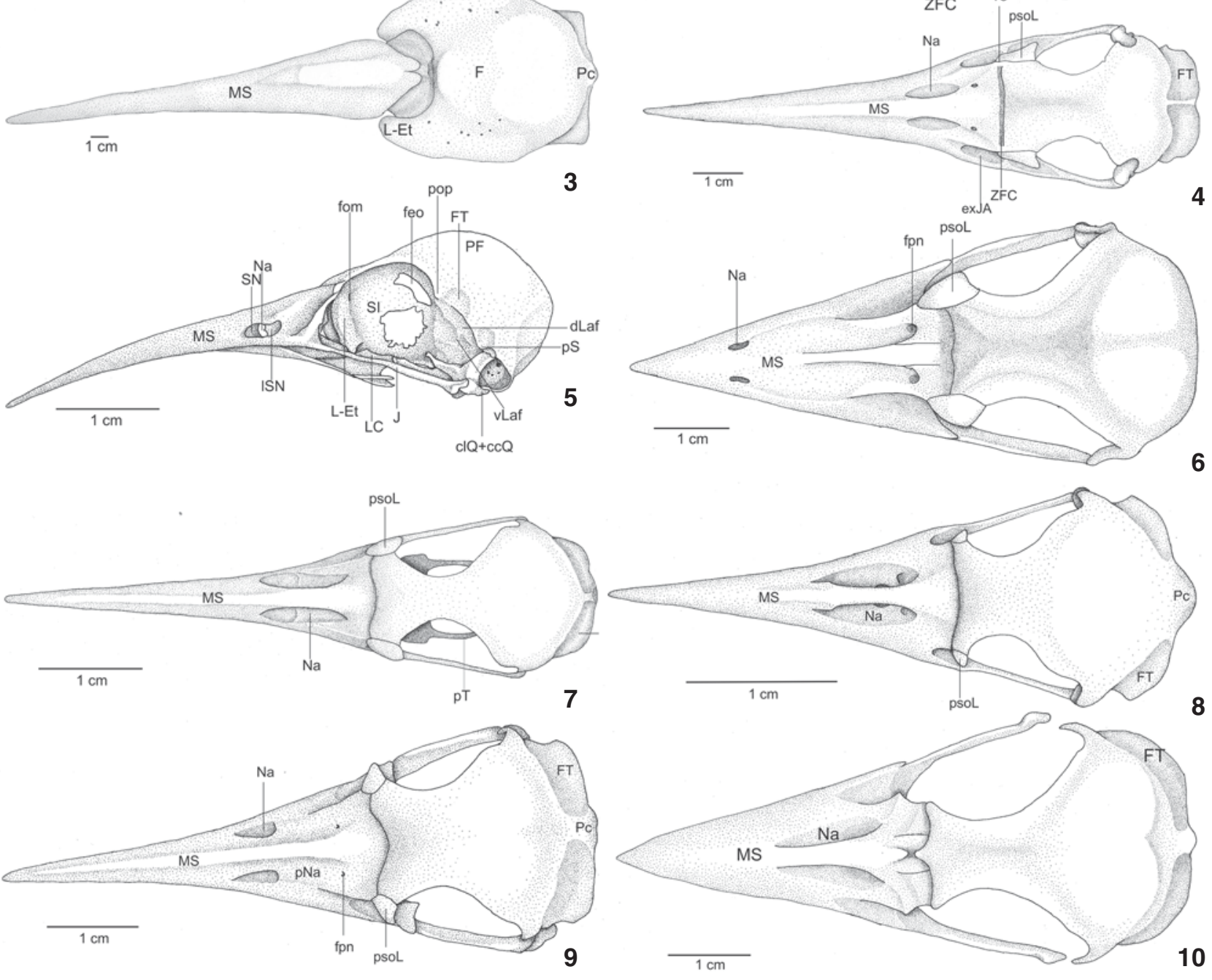

Figuras 1-10. Crânio de: (1) Todus subulatus USNM 555798, vista dorsal; (2) Bucorvus cafer USNM 429091, vista dorsal; (3) Coracias caudata MHNT 134, vista dorsal; (4) Megaceryle torquata USNM 500429, vista dorsal; (5) Phoeniculus purpureus USNM 559060, vista lateral; (6) Leptosomus discolor USNM 291845, vista dorsal; (7) Alcedo atthis MHNT 128, vista dorsal; (8) Merops variegatus USNM 561963, vista dorsal; (9) Nyctyornis amictus USNM 18767, vista dorsal; (10) Brachypteracias leptosomus USNM 223863, vista dorsal. (clQ+ccQ) Fusão dos côndilos lateral e caudal do quadrado, ( $\mathrm{cNa}$ ) concavidade da narina, (dLaf) fossa lateroesfenóide dorsal, (exJA) expansão medial do arco jugal, (F) região frontal, (feo) fenestra orbitocranial, (fom) forame orbitonasal medial, (fpn) forame pós-nasal, (FT) fossa temporal, (J) arco jugal, (LC) lamela coanal do palatino, (L-Et) complexo lacrimal-ectetmóide, (ISN) lâmina óssea paralela ao septo nasal, (MS) maxila superior, (Na) abertura da narina, (Pc) proeminência cerebelar, (PF) Prominentia frontoparietalis, ( $\mathrm{pNa}$ ) placa óssea da abertura da narina, (pop) processo pós-orbital, (pS) processo esquamosal, (psoL) processo supra-orbital do lacrimal, (pT) processo transpalatino, (ro) região ossificada, (SI) septo interorbital, (SN) septo nasal, (vLaf) fossa lateroesfenóide ventral, (ZFC) zona flexória craniofacial.

Revista Brasileira de Zoologia 23 (3): 841-864, setembro 2006 

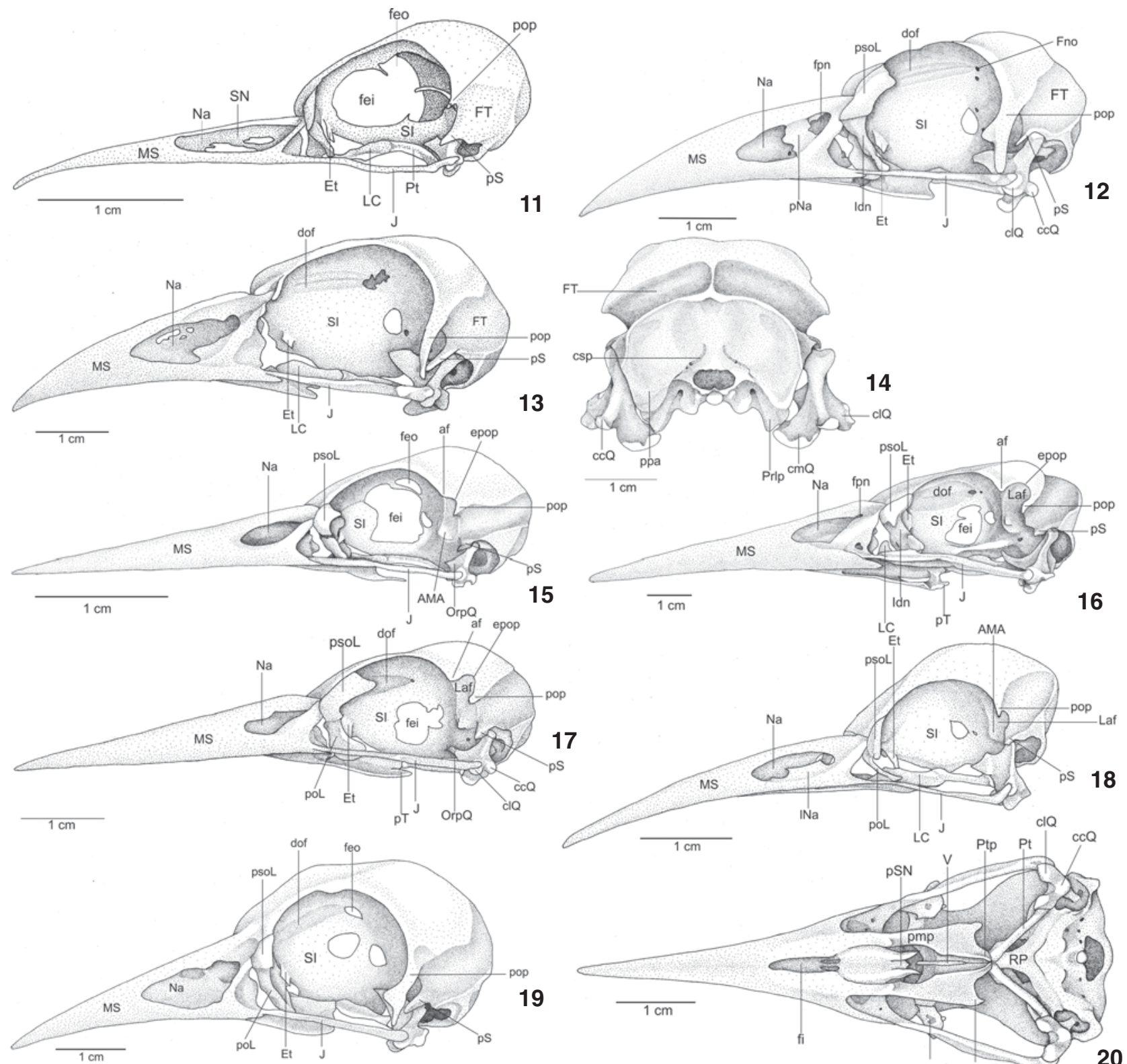

19

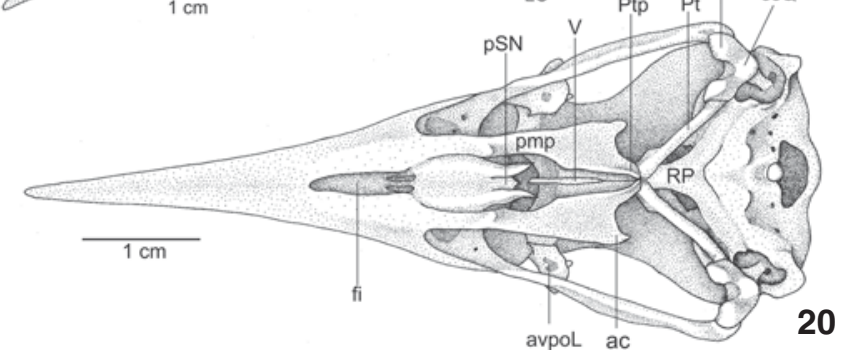

Figuras 11-20. Crânio de: (11) Todus subulatus USNM 555798, vista lateral; (12) Coracias cyanogaster FMNH 320018, vista lateral; (13) Brachypteracias leptosomus USNM 223863, vista lateral; (14) Megaceryle maxima USNM 560529, vista caudal; (15) Ispidina picta UMMZ 223515, vista lateral; (16) Megaceryle maxima USNM 560529, vista lateral; (17) Todirhamphus diops USNM 557193, vista lateral; (18) Merops apiaster USNM 552948, vista lateral; (19) Atelornis pittoides UMMZ 208409, vista lateral; (20) Nyctyornis amictus USNM 18767, vista ventral. (ac) Ângulo caudolateral, (af) ângulo frontal, (AMA) área muscular áspera, (avpoL) abertura da extremidade ventral do processo orbital do lacrimal, (ccQ) côndilo caudal do quadrado, (clQ) côndilo lateral do quadrado, (cmQ) côndilo medial do quadrado, (csp) canal semicircular posterior, (dof) depressão orbitofrontal, (epop) emarginação na borda rostrodorsal do processo pós-orbital, (Et) ectetmóide, (fei) fenestra interorbital, (feo) fenestra orbitocranial, (fi) fenestra interpalatina, (Fno) forame do nervo olfatório, (fpn) forame pós-nasal, (FT) fossa temporal, (Idn) incisura do ducto nasolacrimal, (J) arco jugal, (JA) arco jugal, (Laf) fossa lateroesfenóide, (LC) lamela coanal do palatino, (INa) lâmina da região caudoventral da narina, (MS) maxila superior, (Na) abertura da narina, (OrpQ) processo orbital do quadrado, (pmp) processo maxilar do palatino, (pNa) placa óssea da abertura da narina, (poL) processo orbital do lacrimal, (pop) processo pós-orbital, (ppa) processo paroccipital, (Prlp) processo lateral do paraesfenóide, (pS) processo esquamosal, (pSN) projeção caudal do septo nasal, (psoL) processo supra-orbital do lacrimal, $(\mathrm{pT})$ processo transpalatino, $(\mathrm{pT})$ processo transpalatino, (Pt) pterigóide, (Ptp) processo pterigóideo do palatino, (RP) rostro paraesfenóide, (SI) septo interorbital, (SN) septo nasal, (Sp) processo esquamosal, (V) vômer.

Revista Brasileira de Zoologia 23 (3): 841-864, setembro 2006 
em Coraciidae (pop: Fig. 12) é extremamente desenvolvido (100\%), contactando-se com o arco jugal. Em Leptosomidae há variação individual, sendo que no espécime AMNH 448 o processo pós-orbital ocupa $100 \%$ da distância desde a caixa craniana até o arco jugal, onde contata a região caudolateral do jugal; nos espécimes USNM 291844 e USNM 291845 o processo pós-orbital não alcança o arco jugal, mas encontra-se muito próximo, ocupando cerca de $95 \%$ da distância desde sua origem na caixa craniana até o arco jugal. Dentre os Bucerotidae, o maior desenvolvimento do processo pós-orbital é observado em Buceros vigil e em Buceros bicornis, nos quais ultrapassa a metade da distância até o arco jugal, porém não se contatam. Nas demais espécies ocupa cerca de metade da distância até o arco jugal, com exceção de Tockus fasciatus (Shaw, 1811), T. camurus Cassin, 1857, T. deckeni (Cabanis, 1869) e T. erythrorhynchus (Temminck, 1823) em que é menor, com 1/4 ou menos da distância até o arco jugal. Para Momotidae consulte Pascotto \& Donatelli (2003).

O processo pós-orbital é uma estrutura óssea laminar achatada lateromedialmente, exceto em Leptosomidae em que ele é achatado rostrocaudalmente. Em Bucerotidae verificam-se os dois padrões acima descritos: em Buceros vigil e Buceros rhinoceros Linnaeus, 1758 (espécime fêmea), ele é achatado rostrocaudalmente, ficando a maior amplitude disposta no sentido lateromedial, ao passo que nas demais espécies ele é achatado lateromedialmente. Em Buceros vigil a base dorsal do processo pós-orbital é laminar e há um espessamento na região caudomediana, terminando com a extremidade distal delgada novamente; a margem rostral do processo pós-orbital permanece lisa por toda sua extensão. Já o processo pós-orbital de Buceros rhinoceros (espécime fêmea) difere daquele de Buceros vigil por ser espesso em toda sua extensão, especialmente na base dorsal. Dentre as espécies que apresentam a maior amplitude do processo pósorbital no sentido rostrocaudal, o espécime macho de Buceros rhinoceros e Buceros bicornis têm o processo pós-orbital espesso por toda sua extensão, enquanto que nas demais espécies ele é completamente delgado.

A borda rostrodorsal do processo pós-orbital mostra-se lisa, não havendo formação da emarginação nem do ângulo frontal em Momotidae, Todidae, Meropidae, Coraciidae, Leptosomidae, Brachypteraciidae, Phoeniculidae e Bucerotidae. Em Alcedininae (af, epop: Fig. 15) e Upupidae a emarginação na borda rostrodorsal do processo pós-orbital, bem como o ângulo frontal são reduzidos, enquanto que em Cerylinae (epop: Fig. 16) tanto a emarginação quanto o ângulo frontal (af: Fig. 16) são desenvolvidos. Já em Daceloninae tanto o ângulo frontal (af: Fig. 17) quanto a emarginação (epop: Fig. 17) estão presentes, mas ocorre variação individual no desenvolvimento dessas duas características em Dacelo novaeguineae (Hermann, 1783), Dacelo leachii Vigors \& Horsfield, 1826 e Dacelo gaudichaud Quoy \& Gaimard, 1824, podendo ou não estarem presentes.

O rostro paraesfenóide possui formato ligeiramente triangular, sendo a base caudal ligeiramente mais ampla que a região rostral em Cerylinae, Momotidae, Meropidae (RP: Fig. 20),
Upupidae e Bucerotidae, enquanto que nos demais grupos possui forma levemente retangular, sendo a base caudal e a porção rostral com desenvolvimento semelhantes. Em Alcedininae e Cerylinae (kRP: Fig. 21) ocorre a presença de uma quilha na sua face caudoventral; em Bucerotidae a região rostral do rostro paraesfenóide mostra-se praticamente delgada devido ao maior achatamento lateromedial do rostro paraesfenóide, enquanto que nos demais grupos a região caudoventral do rostro paraesfenóide é espessa e arredondada. O processo basipterigóide é extremamente reduzido ou vestigial em Coraciidae, Leptosomidae, Upupidae e Bucerotidae. Em Alcedininae, Todidae, Meropidae, Brachypteraciidae e Phoeniculidae está ausente, enquanto que em Cerylinae, Daceloninae e Momotidae (Pascotto \& Donatelli 2003) ocorre variação específica, podendo estar ausente em algumas espécies mas, nas espécies em que está presente, é vestigial.

O processo lateral do paraesfenóide apresenta-se extremamente desenvolvido, sendo a extremidade ventral voltada medialmente em Alcedininae, Cerylinae (Prlp: Fig. 14) e Daceloninae; em Bucerotidae (Prlp: Fig. 22) ocorre o menor desenvolvimento, com uma discreta projeção, enquanto que nos demais grupos há um desenvolvimento intermediário. O tubérculo basilar é conspícuo em todos os Coraciiformes. O processo paroccipital é desenvolvido em Cerylinae (ppa: Fig. 14), Daceloninae, Leptosomidae e Upupidae, possuindo desenvolvimento menor nos demais grupos.

A proeminência cerebelar mostra-se extremamente desenvolvida em Todidae (Pc: Fig. 1) e desenvolvida em Leptosomidae e Phoeniculidae, enquanto que em Momotidae, Coraciidae (Pc: Fig. 3), Brachypteraciidae, Upupidae e Bucerotidae (Pc: Fig. 2) é reduzida. Em Meropidae ela é extremamente desenvolvida em Meropogon forsteni e nas espécies de Merops (Pc: Fig. 8), apresentando-se globosa, exceto em Nyctyornis (Pc: Fig. 9) em que é reduzida. Dentre os martins-pescadores (Alcedinidae), ela é reduzida na maioria das espécies, mas em Megaceryle maxima (Fig. 14), M. lugubris e M. torquata (Cerylinae), Pelargopsis capensis e $P$. melanorhyncha (Daceloninae) ela está praticamente ausente, conferindo um aspecto plano à região supra-occipital. Em Megaceryle alcyon (Cerylinae) há variação individual, estando ausente nos espécimes USNM 553851 e USNM 621370, enquanto que nos demais espécimes é reduzida. A concavidade da região supraoccipital, na maioria das espécies de Coraciiformes, é rasa, exceto em Bucerotidae em que é praticamente plana. Dentre os martinspescadores das subfamílias Cerylinae e Daceloninae ocorre variação específica, sendo em Megaceryle maxima (Fig. 14), M. torquata, M. lugubris (Cerylinae), Pelargopsis capensis e P. melanorhyncha (Daceloninae) plana e, nas demais espécies, com uma discreta profundidade.

O canal semicircular posterior está ausente em Bucerotidae (Fig. 22). Ele se apresenta reduzido e situado lateralmente ao forame magno em Meropidae, Leptosomidae, Phoeniculidae e Upupidae; em Coraciidae e Brachypteraciidae ele se situa dorsolateralmente ao forame magno. Nos martinspescadores da subfamília Alcedininae, ele estende-se acima do 
forame magno em Alcedo azurea, A. atthis, A. cyanopecta, Ceyx rufidorsa, C. melanurus, C. erithacus, C. lepidus, Ispidina lecontei e I. picta, enquanto que em A. cristata, A. websteri, A. quadribrachys, A. leucogaster e $A$. semitorquata ele se dispõe lateroventralmente ao forame magno, mas sem se projetar dorsalmente. O canal semicircular posterior não foi comparado com C. fallax por estar danificado. Já nos Cerylinae dispõe-se dorsolateralmente ao forame magno em Megaceryle maxima (csp: Fig. 14), M. lugubris, M. torquata, M. alcyon, Chloroceryle amazona (Latham, 1790), C. americana, C. inda (Linnaeus, 1766) e C. aenea, enquanto que em Ceryle rudis situa-se lateralmente. Nos Daceloninae, ele possui desenvolvimento menor, ocupando apenas uma reduzida porção lateroventral ao forame magno em Pelargopsis capensis e $P$. melanorhyncha, ao contrário das demais espécies em que tem maior desenvolvimento, estendendo-se ligeiramente acima do forame magno. Já em Todidae e Momotidae (PAscotto \& Donatelli 2003: Fig. 4) o canal semicircular posterior apresenta-se desenvolvido, estendendo-se acima da margem dorsal do forame magno. Em Merops variegatus e M. pusillus (Meropidae) não há evidências osteológicas da presença do canal semicircular posterior.

\section{Orbita}

A condição típica do lacrimal (Os lacrimale), formado pelos processos supra-orbital (Proc. supraorbitalis) e orbital (Proc. orbitalis), está ausente em Momotidae (Pascotto \& Donatelli 2003: figs 1-3, 6-10) e nos indivíduos adultos de Upupidae, Phoeniculidae e Bucerotidae. No entanto, a análise de indivíduos jovens de calaus [Buceros rhinoceros, B. bicornis e Ceratogymna brevis (Friedmann, 1929)] revela que o lacrimal (L: Fig. 23) encontra-se presente e distinto do ectetmóide (Et: Fig. 23), mas eles se fundem completamente nos indivíduos adultos (LEt: Fig. 2), podendo o mesmo ocorrer nos indivíduos adultos de Upupidae e Phoeniculidae. Nos demais grupos a condição típica do lacrimal está presente. O processo supra-orbital do lacrimal articula-se medialmente com a margem lateral dos ossos frontal e nasal por meio da Facies articularis frontonasalis e ocupa cerca de $30 \%$ ou menos da distância desde sua porção mais rostral até a região dorsocaudal da órbita em Alcedininae (psoL: Figs 7, 15), Cerylinae (psoL: Figs 4, 16), Meropidae (psoL: Figs 8, 9, 18), Todidae (psoL: Fig. 1), Coraciidae (psoL: Figs 3, 12), Brachypteraciidae (psoL: Fig. 19), Leptosomidae (psoL: Fig. 6) e na maioria das espécies de Daceloninae (psoL: Fig. 17), exceto em Dacelo novaeguineae (psoL: Fig. 24), D. leachii, D. gaudichaud, Syma torotoro Lesson, 1827 e Melidora macrorrhina (Lesson, 1827) em que se mostra longo e pontudo, possuindo o maior desenvolvimento dentre todos os Coraciiformes, com cerca de $70 \%$ da distância desde sua porção mais rostral até a região caudal da órbita.

O processo orbital do lacrimal contata-se ventrolateralmente com o arco jugal (poL: Figs 17, 18, 19). Em Alcedininae, Cerylinae, Daceloninae, Todidae, Coraciidae e Brachypteraciidae o processo orbital do lacrimal é amplo e articula-se ou contatase com a porção distal do ectetmóide, enquanto que em Meropidae (poL: Fig. 18) e Leptosomidae não há contato entre essas duas estruturas. Em Coraciidae a margem dorsomedial do processo orbital encontra-se extremamente desenvolvida, quase que fechando completamente a comunicação entre a órbita e a fenestra anteorbital; em Cerylinae, Todidae e Brachypteraciidae a margem dorsomedial do processo orbital é desenvolvida, porém menor, havendo a formação de uma pequena área de comunicação. O processo orbital do lacrimal de Meropidae (poL: Fig. 18) e Leptosomidae apresenta-se estreito, não obstruindo tal comunicação. Em Daceloninae, a expansão dorsal da margem dorsomedial do processo orbital é extremamente desenvolvida em Melidora macrorrhina, Dacelo novaeguineae, D. leachii, $D$. gaudichaud, Actenoides lindsayi (Vigors, 1831), Tanysiptera galatea Gray, 1859 e T. sylvia Gould, 1850, quase tocando o septo interorbital medialmente e o osso frontal dorsalmente; em Actenoides lindsayi, T. galatea e T. sylvia a projeção dorsomedial do processo orbital do lacrimal tem ainda um entalhe com profundidade variável que permite a comunicação com a fenestra anteorbital. Ela é reduzida em Pelargopsis capensis, $P$. melanorhyncha, Halcyon malimbica (Shaw, 1811) e Todirhamphus winchelli; nas demais espécies ela tem desenvolvimento intermediário. Em H. badia Verreaux \& Verreauxi, 1851 observa-se variação individual. Já nos Alcedininae tal expansão é desenvolvida em Alcedo leucogaster, A. cristata, A. cyanopecta, Ceyx lepidus, C. fallax, Ispidina lecontei e I. picta, enquanto que em Alcedo quadribrachys, A. meninting, A. azurea, A. websteri, A. semitorquata, Ceyx erithacus, $C$. rufidorsa e $C$. melanurus a expansão dorsomedial é menor. Em A. atthis ocorre variação individual, podendo existir as duas condições acima descritas.

Na borda lateral do processo orbital do lacrimal destacase a incisura do ducto nasolacrimal (Incisura ductus nasolacrimalis), uma emarginação ampla e rasa em Alcedininae, Leptosomidae e Brachypteraciidae e com profundidade ligeiramente maior em Cerylinae (Idn: Fig. 16). Em Todidae e Meropidae (poL: Fig. 18) a borda lateral do processo orbital do lacrimal é reta, não havendo diferenciação da incisura do ducto nasolacrimal. Dentre os Daceloninae, ela é ampla e rasa em Halcyon coromanda (Latham, 1790), H. smyrnensis (Linnaeus, 1758), H. pileata (Boddaert, 1783), H. malimbica e Todiramphus winchelli, mas nas demais espécies ela possui menor amplitude e maior profundidade. Em Tanysiptera galatea ocorre variação individual. Em Coraciidae, a incisura do ducto nasolacrimal é ampla e rasa nas espécies de Coracias Linnaeus, 1758 (Idn: Fig. 12), mas apresenta variação individual na amplitude em $C$. noevia Daudin, 1800. Nas espécies de Eurystomus Vieillot, 1816 ela tem maior profundidade e menor amplitude, mas há variação individual em E. azureus e E. gularis Vieillot, 1819.

A porção ventral do processo orbital tem aspecto bulboso em Alcedininae, Cerylinae, Todidae, Coraciidae e Brachypteraciidae, diferentemente de Meropidae em que é delgada, evidenciando ainda uma pequena abertura ou forame (avpoL: Fig. 20). Já em Leptosomidae ela é reta, porém levemente espessa. $\mathrm{Na}$ maioria das espécies de Daceloninae ela também tem aspecto bulboso, mas em Dacelo novaeguineae, D. leachii, D. 

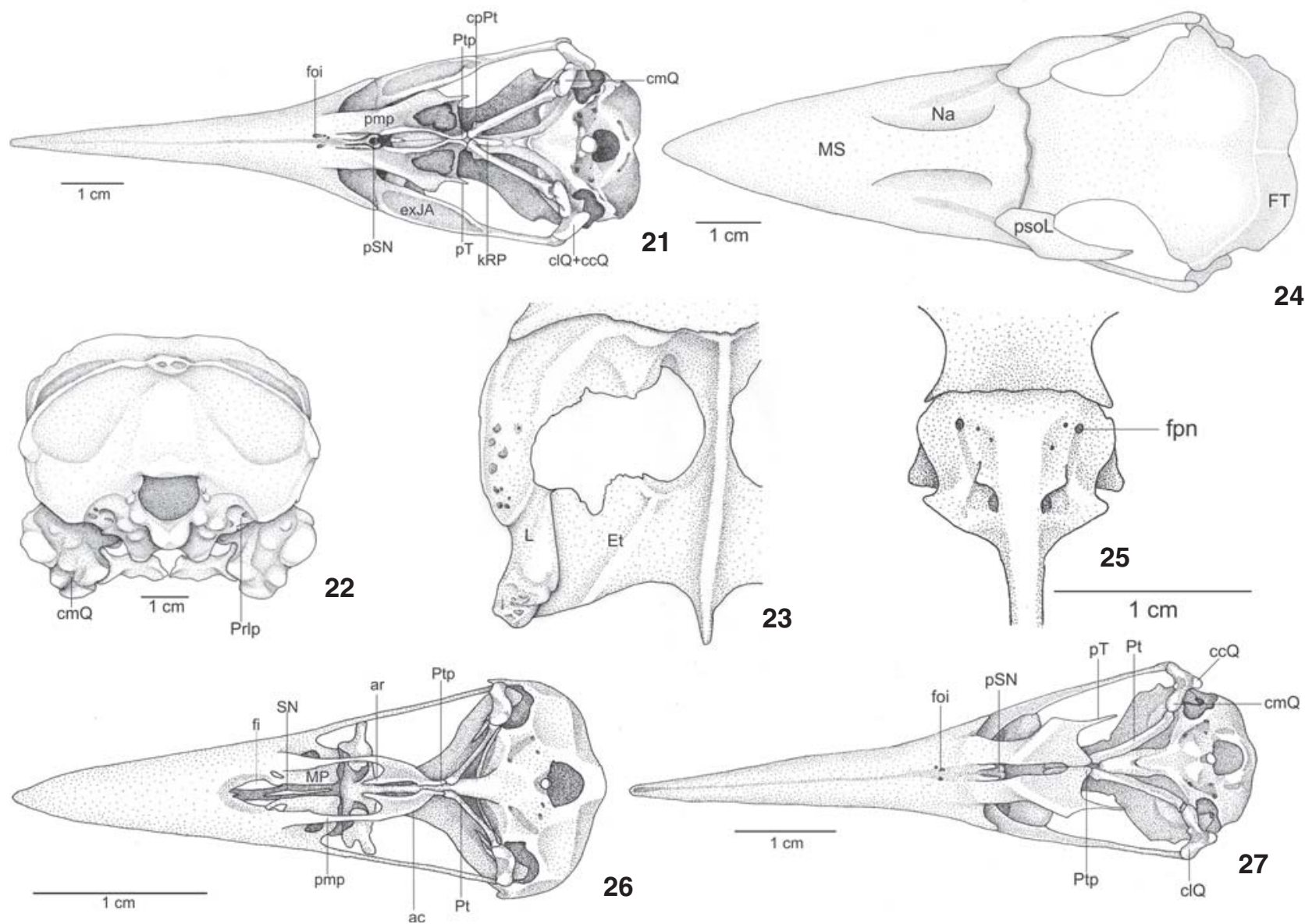

Figuras 21-27. Crânio de: (21) Megaceryle lugubris USNM 318238, vista ventral; (22) Bucorvus cafer USNM 288484, vista caudal; (23) Ceratogymna brevis USNM 488832, vista frontal do lacrimal e ectetmóide de um espécime jovem (sem escala); (24) Dacelo novaeguineae MHNT 126, vista dorsal; (25) Rhinopomastus minor UKMNH 70670, vista dorsorrostral da região caudal da maxila superior; (26) Todus subulatus USNM 555798, vista ventral; (27) Alcedo semitorquata UMMZ 158188, vista ventral. (ac) Ângulo caudolateral, (ar) ângulo rostromedial, (ccQ) côndilo caudal do quadrado, (clQ) côndilo lateral do quadrado, (clQ+ccQ) fusão dos côndilos lateral e caudal do quadrado, (cmQ) côndilo medial do quadrado, (cpPt) crista rostrolateral do pterigóide, (Et) ectetmóide, (exJA) expansão medial do arco jugal, (fi) fissura interpalatina, (foi) forame interpalatino, (fpn) forame pós-nasal, (FT) fossa temporal, (kRP) quilha da face caudoventral do rostro paraesfenóide, (L) lacrimal, (MP) processo maxilopalatino, (MS) maxila superior, (Na) abertura da narina, (pmp) processo maxilar do palatino, (Prlp) processo lateral do paraesfenóide, (pSN) projeção caudal do septo nasal, (psoL) processo supra-orbital do lacrimal, (pT) processo transpalatino, (Pt) pterigóide, (Ptp) processo pterigóideo do palatino, (SN) septo nasal.

gaudichaud e Melidora macrorrhina ela é laminar e levemente curvada caudalmente.

O ectetmóide e o lacrimal estão fundidos nos adultos de Bucerotidae (L-Et: Fig. 2) e, possivelmente, em Phoeniculidae (LEt: Fig. 5) e Upupidae, formando o complexo lacrimal-ectetmóide. Este se mostra como uma grande placa que praticamente obstrui a comunicação da órbita com a fenestra anteorbital. Nesses três grupos há a formação do típico Foramen orbitonasale mediale, que é extremamente reduzido em Upupidae e Phoeniculidae (fom: Fig. 5). Já em Bucerotidae ele é extremamente desenvolvido em Bucorvus abyssinicus, Bucorvus cafer e no espécime fêmea de Aceros plicatus (Forster, 1781), nos quais ocupa quase toda a região rostrodorsal da órbita; é pequeno nas espécies de Tockus Lesson, 1830 e em Ceratogymna elata (Temminck, 1831), e desenvolvido nas demais espécies de calaus. Nos indivíduos jovens de Buceros bicornis e B. rhinoceros o forame orbitonasal medial possui desenvolvimento maior em relação aos espécimes adultos, certamente devido ao maior grau de ossificação do crânio nos adultos. Apesar do lacrimal típico estar ausente em Momotidae (Pascotto \& Donatelli 2003: figs 6, 7, 9, 10), o ectetmóide mantém-se reduzido, diferentemente de Upupidae, Phoeniculidae e Bucerotidae. Nos demais grupos em que

Revista Brasileira de Zoologia 23 (3): 841-864, setembro 2006 
é distinto do lacrimal, o ectetmóide mede cerca de 1/3 da distância desde o mesetmóide até o arco jugal em Leptosomidae e Brachypteraciidae (Et: Figs 13, 19), enquanto que em Alcedininae, Todidae (Et: Fig. 11), Cerylinae (Et: Fig. 16), Daceloninae (Et: Fig. 17), Momotidae e Meropidae (Et: Fig. 18) é mais longo, com cerca de metade da distância do mesetmóide ao arco jugal. Dentre os Coraciidae, o ectetmóide é cerca de duas vezes mais longo e estreito nas espécies de Coracias (Et: Fig. 12) que em Eurystomus, nas quais ele é mais curto e mais largo dorsoventralmente. Ainda, o maior desenvolvimento do ectetmóide é observado em Coracias spatulata Trimen, 1880, alcançando mais da metade da distância desde sua base no mesetmóide até o arco jugal.

Nos grupos em que está distinto, o ectetmóide projeta-se lateralmente em Leptosomidae, enquanto que em Todidae (Et: Fig. 11), Coraciidae (Et: Fig. 12), Alcedininae, Cerylinae (Et: Fig. 16), Daceloninae, Meropidae (Et: Fig. 18), Momotidae e Brachypteraciidae (Et: Figs 13,19) projeta-se lateroventralmente a partir da região mesetmóide.

O septo interorbital apresenta-se totalmente ossificado em Upupidae, Phoeniculidae [SI: Fig. 5 (neste espécime a região está danificada)], Coraciidae (SI: Fig. 12), Meropidae (SI: Fig. 18) e na maioria das espécies de Bucerotidae, exceto em Buceros vigil em que se encontra completamente ossificado. Em Todidae (SI: Fig. 11), Alcedininae (SI: Fig. 15), Cerylinae (SI: Fig. 16), Daceloninae (SI: Fig. 17) e Leptosomidae ele é parcialmente ossificado, havendo a formação da fenestra interorbital (fei: Figs 11, 15-17). Em Brachyptraciidae e Momotidae ocorrem variações específicas, sendo que em Brachypteraciidae está totalmente ossificado em Brachypteracias leptosomus (SI: Fig. 13) e parcialmente ossificado em Atelornis pittoides (SI: Fig. 19) e A. crossleyi. Para as variações de Momotidae, vide Pascotto \& Donatelli (2003).

O forame olfatório é distinto e reduzido em Momotidae, Meropidae, Coraciidae (Fno: Fig. 12), Leptosomidae e Bucerotidae, enquanto que em Cerylinae e Daceloninae ele se mostra com abertura em grau variado entre as espécies. Em Alcedininae, Todidae, Brachypteraciidae, Phoeniculidae e Upupidae o forame olfatório encontra-se fundido com o fontículo orbitocranial, formando a fenestra orbitocranial (feo: Figs 5, 11, 15, 19). Entretanto, ocorre diferença no desenvolvimento dessa fenestra: ela é pequena em Alcedininae (feo: Fig. 15) e Brachypteraciidae (feo: Fig. 19), desenvolvida em Upupidae e extremamente desenvolvida em Phoeniculidae (feo: Fig. 5) e Todidae (feo: Fig. 11). Em Phoeniculidae (feo: Fig. 5) a fenestra orbitocranial tem forma retangular, sendo aproximadamente duas vezes mais longa rostrocaudalmente que ampla dorsoventralmente e estende-se lateralmente pela região lateroesfenóide. Já em Upupidae ela tem uma forma arredondada e ocupa o ângulo dorsocaudal da região orbital, estendendo-se por uma pequena porção dorsomedial da região lateroesfenóide.

Na região rostrodorsal da cavidade orbital encontra-se a depressão orbitofrontal. Ela é rasa e curta e está restrita à região rostral do septo interorbital em Alcedininae, e rasa e longa, ocupando toda a extensão do sulco olfatório em Coraciidae (dof: Fig. 12), Brachypteraciidae (dof: Figs 13, 19), Cerylinae (dof: Fig. 16), Daceloninae (dof: Fig. 17) e Momotidae. Na maioria das espécies de Meropidae (Fig. 18) e de Bucerotidae a depressão orbitofrontal está ausente, sendo a região plana, exceto em Nyctyornis amictus (Meropidae) em que é ampla e está restrita à região rostrodorsal do septo interorbital e, dentre os Bucerotidae, em Aceros comatus (Raffles, 1822), Bucorvus abyssinicus e Bucorvus cafer em que é rasa e longa, ocupando toda a extensão do sulco olfatório. Nos demais grupos ela está ausente, sendo a região praticamente plana.

A área muscular áspera é desenvolvida e globosa em Alcedininae (AMA: Fig. 15), Cerylinae (Fig. 16), Daceloninae e Momotidae; extremamente reduzida em Phoeniculidae (Fig. 5), Meropidae (AMA: Fig. 18), Leptosomidae e Upupidae, enquanto que em Todidae (Fig. 11) ela é parcialmente ossificada. Em Brachypteraciidae ela é reduzida em Brachypteracias leptosomus e Atelornis crossleyi, sendo pouco mais desenvolvida em $A$. pittoides, enquanto que nos Coraciidae é extremamente reduzida nas espécies de Coracias, diferentemente de Eurystomus em que a região é praticamente plana. Já nos Bucerotidae a área muscular áspera é desenvolvida e globosa nas espécies de Tockus e Penelopides, enquanto que nas demais espécies é reduzida, mas em algumas espécies torna-se difícil sua diferenciação em virtude do desenvolvimento do processo orbitoesfenoidal. Com relação ao processo orbitoesfenoidal, ele está ausente em Phoeniculidae (Fig. 5), Todidae (Fig. 11), Alcedininae (Fig. 15), Meropidae (Fig. 18), Coraciidae, Brachypteraciidae e Upupidae. Em Leptosomidae ele está presente, mas é curto e levemente espesso, com aspecto triangular, enquanto que em Cerylinae é inconspícuo. Entre os Bucerotidae, o processo orbitoesfenoidal está ausente nas espécies de Tockus e Penelopides, sendo nas demais espécies pequeno ou vestigial. Já o maior desenvolvimento e espessura do processo orbitoesfenoidal é observado, dentre os Coraciiformes, nas espécies de Buceros Linnaeus, 1758 e de Bucorvus (Bucerotidae). Para as variações de Momotidae vide Pascotto \& Donatelli (2003).

A fossa lateroesfenóide situa-se na porção lateral da região lateroesfenóide e é rasa e relativamente longa, estendendo-se dorsoventralmente até a margem caudolateral da região frontal, rostralmente ao processo pós-orbital em Alcedininae, Cerylinae (Laf: Fig. 16), Daceloninae (Laf: Fig. 17), Meropidae (Laf: Fig. 18), Todidae, Upupidae e Bucerotidae; é extremamente rasa e ampla, ocupando toda a porção lateral da região lateroesfenóide em Brachypteraciidae. Já em Leptosomidae e Coraciidae ela está reduzida à porção lateroventral da região lateroesfenóide, lateralmente à área muscular áspera, com forma ligeiramente oval. Em Phoeniculidae (Fig. 5) a fossa lateroesfenóide é rasa mas apresenta uma conformação única, pois é dividida em duas fossas: uma dorsolateral (dLaf: Fig. 5), com formato ligeiramente retangular e disposta dorsoventralmente, imediatamente rostromedial ao processo pós-orbital; e outra ventral (vLaf: Fig. 5), apresentando-se oval e mais cons- 
pícua que a porção dorsal e deslocada ventromedialmente em relação à fossa dorsal. As duas porções da fossa lateroesfenóide são separadas por uma conspícua crista, além de haver um certo desnível entre elas. A diferenciação da fossa lateroesfenóide somente é visível a olho nú em Phoeniculus Jarocki 1821 em virtude do maior tamanho do crânio, mas quando observado com o auxílio de um estereomicroscópio também é possível identificá-la nas espécies de Rhinopomastus Jardine 1828.

\section{Ossa Maxillae}

A maxila superior corresponde a cerca de $50 \%$ a $60 \%$ do comprimento total do crânio em Todidae (MS: Fig. 1), Coraciidae (MS: Fig. 3), Cerylinae (MS: Fig. 4), Leptosomidae (MS: Fig. 6), Alcedininae (MS: Fig. 7), Meropidae (MS: Figs 8, 9), Brachypteraciidae (MS: Fig. 10), Daceloninae (MS: Fig. 24) e Momotidae, e cerca de 70\% em Phoeniculidae, Upupidae e Bucerotidae (MS: Fig. 2). A maxila superior apresenta uma leve curvatura ventral (MS: Figs 11, 13, 18, 19), exceto em Upupidae e Bucerotidae em que é mais acentuada. Dentre os Coraciidae há variação, sendo que nas espécies de Coracias (MS: Fig. 12) a curvatura ventral assemelha-se ao padrão de Momotidae (Pascotto \& Donatelli 2003), enquanto que nas espécies de Eurystomus a curvatura ventral é terminal e ligeiramente mais acentuada. Nos Phoeniculidae a curvatura ventral é discreta em Phoeniculus purpureus (Miller, 1784) (MS: Fig. 5) e P. bollei (Hartlaub, 1858), enquanto que em Rhinopomastus cyanomelas (Vieillot, 1819) e R. minor (Rüppell, 1845) é acentuada, semelhante ao padrão de Upupidae e Bucerotidae. Em Alcedininae (MS: Fig. 15), Cerylinae (MS: Fig. 16) e Daceloninae (MS: Fig. 17) a maxila superior é praticamente reta.

A maxila superior é espessa, sendo a base caudal cerca de 1,4 vez mais ampla que alta em Cerylinae (MS: Figs 4, 16) e Brachypteraciidae (MS: Figs 10, 13, 19); cerca de 1,7 vez em Phoeniculidae (MS: Fig. 5), Leptosomidae (MS: Fig. 6), Alcedininae (MS: Figs 7, 15) e Upupidae; em Todidae (MS: Figs 1, 11) apresenta-se delgada, sendo aproximadamente 2,5 vezes mais ampla do que alta na sua base. Em Meropidae ocorre variação, sendo cerca de três vezes mais ampla que alta em Merops hirundinaceus e $M$. pusillus; cerca de 2,5 vezes em $M$. orientalis, $M$. philippinus, M. ornatus, M. albicollis, M. superciliosus, M. malimbicus, M. apiaster e M. bulocki; e cerca de duas vezes em Nyctyornis amictus (MS: Fig. 9), N. athertoni, M. bullockoides, M. nubicus, M. muelleri, M. oreobates, M. variegatus (MS: Fig. 8) e M. gularis (não comparável com Meropogon forsteni por estar danificada no único exemplar disponível para estudo). Na maioria das espécies de Daceloninae, sua base caudal é cerca de 1,7 vez a duas vezes mais ampla que alta, exceto em Pelargopsis capensis e $P$. melanorhyncha em que é mais estreita e alta, excedendo cerca de 1,4 vez a sua altura. Nos Coraciidae também há variação, sendo a base caudal da maxila superior cerca de 1,4 vez mais ampla que alta nas espécies de Coracias, enquanto que em Eurystomus é cerca de duas vezes mais ampla que alta. Dentre os Momotidae, é cerca de 1,4 vez mais ampla que alta nas espécies de Momotus Brisson, 1760 e de Baryphthengus Cabanis \& Heine, 1859; cerca de 1,7 vez em Aspatha gularis (Lafresnaye, 1840), Hylomanes momotula (Lichtenstein, 1839) e Electron carinatum (Du Bus de Gisignies, 1847), sendo a maior amplitude observada em Eumomota superciliosa (Sandbach, 1837) (duas vezes) e em Electron platyrhynchum (Leadbeater, 1829) (2,5 vezes). Nos Bucerotidae a maxila superior é visivelmente mais alta dorsoventralmente que ampla lateromedialmente, mas a existência da Galea ossea não permitiu medir suas dimensões exatas.

A abertura externa da narina é ampla e oval, ocupando cerca de $40 \%$ do comprimento da maxila superior em Todidae (Na: Figs 1, 11) e Brachypteraciidae (Na: Figs 10, 13, 19); é reduzida em Coraciidae (Na: Figs 3, 12), Cerylinae (Na: Figs 4, 16) e Alcedininae (Na: Figs 7, 15), ocupando cerca de $20 \%$ da maxila superior, e cerca de 15\% em Leptosomidae (Na: Fig. 6); é extremamente reduzida em Phoeniculidae (Na: Fig. 5), Upupidae e Bucerotidae, nos quais ocupa cerca de $5 \%$ do comprimento da maxila superior. Dentre os Meropidae, em Merops Linnaeus, 1758 (INa: Fig. 18) e em Meropogon Bonaparte, 1850, a abertura externa da narina é margeada por uma lâmina conspícua que se projeta dorsalmente a partir de sua borda caudoventral, mas esta não alcança a borda dorsal da abertura da narina e, conseqüentemente, não obstrui sua abertura. Já em Nyctyornis Jardine \& Selby, 1830 (pNa: Fig. 9) essa lâmina é desenvolvida e em forma de uma placa, obstruindo cerca de $25 \%$ da abertura da narina, semelhante ao observado em Coraciidae (pNa: Figs 3, 12). O desenvolvimento da abertura da narina também é variável nos Meropidae: ocupa cerca de $40 \%$ do comprimento da maxila superior em M. muelleri; cerca de 30\% em Meropogon forsteni, Merops bullockoides, M. albicollis, M. oreobates, M. orientalis, M. variegatus (Na: Fig. 8), M. gularis, M. malimbicus; cerca de 25\% em M. bulocki, M. hirundinaceus, M. apiaster (Na: Fig. 18), M. pusillus, M. superciliosus, M. nubicus, M. philippinus e M. ornatus; $20 \%$ em N. athertoni; e $10 \%$ em N. amictus (Na: Fig. 9). Nos martins-pescadores da subfamília Daceloninae, a abertura externa da narina (Na: Figs 17, 24) tem forma oval, exceto em Pelargopsis capensis e $P$. melanorhyncha em que é estreita e longa. Ela ocupa cerca de $30 \%$ do comprimento da maxila superior na maioria das espécies, exceto em Melidora macrorrhina, Pelargopsis capensis e $P$. melanorhyncha em que se mostra menor, com cerca de $15 \%$ a $20 \%$ do comprimento da maxila superior. Em Momotidae também ocorre variação específica quanto ao seu desenvolvimento (Pascotto \& Donatelli 2003).

A abertura externa da narina situa-se na porção caudolateral da maxila superior, exceto em Leptosomidae (Na: Fig. 6) em que se abre na porção rostrolateral da maxila superior. Apesar de nos Bucerotidae ela também ser caudolateral, diferenciase dos demais grupos por ocupar uma posição mais dorsal em relação à orbita. A concavidade da narina tem a forma de uma fenda, sendo estreita e profunda em Alcedininae e Cerylinae; mostra-se rasa e ampla em Daceloninae, Todidae (cNa: Fig. 1), Meropidae e Brachypteraciidae. Em Leptosomidae, Phoeniculidae e Upupidae não é possível identificar a concavidade da narina em virtude da presença da placa óssea que recobre qua- 
se toda a abertura da narina. Já em Coraciidae a concavidade da narina está ausente, enquanto que em Momotidae (PAscotto \& Donatelli 2003) ocorre variação específica; não comparável com Bucerotidae em função do maior grau de ossificação e fusão dos ossos da maxila superior.

O forame pós-nasal apresenta-se fundido com a abertura da narina em Todidae (Na: Fig. 1), Alcedininae (Na: Fig. 7), Brachypteraciidae (Na: Fig. 10), Daceloninae (Na: Fig. 24) e Momotidae, e distinto da abertura da narina em Leptosomidae (fpn: Fig. 6), Coraciidae (fpn: Fig. 12), Phoeniculidae (fpn: Fig. 25), Upupidae e Bucerotidae. Nas espécies de Cerylinae também é distinto, mas ocorre variação individual em Megaceryle maxima (fpn: Fig. 16) e Chloroceryle amazona, podendo estar fundido com a abertura da narina em alguns espécimes. Em Meropidae o forame pós-nasal funde-se com a abertura da narina em Meropogon forsteni e nas espécies de Merops (Na: Fig. 8), mas em N. amictus (fpn: Fig. 9) e N. athertoni ele permanece distinto e é extremamente reduzido.

O septo nasal é laminar e delgado, sendo completamente ossificado em Alcedininae (Fig. 15) e Daceloninae (Fig. 17); em Todidae (SN: Fig. 11) apresenta grau de ossificação reduzido. Já em Coraciidae e Leptosomidae não é possível identificálo totalmente, devido à presença da placa óssea que recobre a abertura da narina externamente. Em Cerylinae (Fig. 16) e Meropidae (Fig. 18) pode haver alguma variação individual, mas na maioria das espécies encontra-se totalmente ossificado. Em Phoeniculidae (SN: Fig. 5) e Upupidae o septo nasal é delgado, mas evidenciam-se duas lâminas ósseas (ISN: Fig. 5) dispostas paralela e intimamente em contato com o septo nasal, obliterando-o quase que por completo. Em Bucerotidae o septo nasal é totalmente ossificado e indistinto da maxila superior, enquanto que em Brachypteraciidae ele tem grau de ossificação reduzido em Brachypteracias leptosomus (Fig. 13) e Atelornis pittoides e está completamente ossificado em A. crossleyi. Em Momotidae também há variação (Pascotto \& Donatelli 2003).

Os processos maxilopalatinos de Todidae (MP: Fig. 26) não se encontram fundidos medialmente, havendo um espaço estreito entre eles, onde se nota a face ventral do septo nasal (SN: Fig. 26) medialmente. Nos demais grupos eles se fundem medialmente, mas em Bucerotidae estão indistintos e quase que totalmente encobertos e fundidos numa estrutura única com os demais ossos da maxila superior.

Nos grupos em que os processos maxilopalatinos estão fundidos entre si e com a face ventral do septo nasal, a projeção caudal do septo nasal é ligeiramente mais curta que a projeção caudal do maxilopalatino em Momotidae, Meropidae (pSN: Fig. 20) e Coraciidae, sendo nesta última cerca de três a quatro vezes menor. Já em Upupidae e Phoeniculidae a projeção caudal do septo nasal é maior que a projeção caudal do maxilopalatino. Em Alcedininae, ela é maior que a projeção caudal do maxilopalatino em Alcedo azurea, A. websteri, A. quadribrachys, A. atthis, A. leucogaster, A. semitorquata (pSN: Fig. 27) e A. cyanopecta; em A. cristata, Ceyx lepidus, C. erithacus, C. rufidorsa,
C. melanurus, C. fallax, Ispidina lecontei e I. picta possuem desenvolvimento semelhantes ou a projeção caudal do septo nasal pode ser menor que a do maxilopalatino. Nos Cerylinae ela é reduzida em Megaceryle alcyon e Ceryle rudis, não ultrapassando o comprimento da projeção caudal do maxilopalatino, enquanto que nas demais espécies ela é desenvolvida, cujo comprimento é pouco maior que o da projeção caudal do maxilopalatino (pSN: Fig. 21). Dentre os Daceloninae, ela possui comprimento maior que a projeção caudal do maxilopalatino em Pelargopsis capensis, P. melanorhyncha e Halcyon pileata, enquanto que nas demais espécies ela possui comprimento igual ou menor que a projeção caudal do maxilopalatino. Em Brachypteraciidae a projeção caudal do septo nasal é pouco mais longa que a projeção caudal do maxilopalatino nas espécies de Atelornis Pucheran, 1846 que em Brachypteracias leptosomus, na qual a projeção caudal do septo nasal é mais curta. Não comparável com Bucerotidae pela completa fusão dos ossos da maxila superior em uma unidade única, bem como com Leptosomidae por estar completamente fundida com os processos maxilopalatinos.

Na região caudoventral da maxila superior, local de fusão dos processos maxilares do palatino e também de parte dos maxilopalatinos, ocorre a formação de dois diminutos foramens, denominados de forames interpalatinos (novo termo), presentes em Cerylinae (foi: Fig. 21), Alcedininae (foi: Fig. 27) e Daceloninae; em Meropidae (fi: Fig. 20), Todidae (fi: Fig. 26), Coraciidae, Leptosomidae, Brachypteraciidae, Phoeniculidae e Upupidae a abertura é maior, formando a fissura interpalatina. Em Bucerotidae tanto os foramens quanto a fissura interpalatina estão ausentes, sendo a região completamente ossificada.

Palati

O processo maxilar do palatino (pmp: Figs 20, 21, 26) é longo, com a porção rostral mais estreita que a caudal, exceto em Momotidae (Pascotto \& Donatelli 2003: Figs 11, 12, 13) em que se mostra com a mesma amplitude por toda sua extensão. Em Bucerotidae o processo maxilar do palatino encontra-se fundido por quase toda sua extensão com a maxila superior, sendo distinta somente a porção caudal, a partir da região do ângulo caudomedial. Em Daceloninae, o processo maxilar do palatino é estreito por toda sua extensão em Dacelo novaeguineae, D. leachii, D. gaudichaud, Melidora macrorrhina, Pelargopsis capensis, P. melanorhyncha, Halcyon coromanda, $H$. senegalensis (Linnaeus, 1766) e Tanysiptera galatea, enquanto que nas demais espécies a porção caudal é mais ampla que a porção rostral. A partir da região mediana, a Pars lateralis expande-se lateromedialmente, bem como apresenta uma torção em seu eixo longitudinal, ficando a margem lateral posicionada ventralmente em relação à margem medial, sendo que a inclinação do palatino tem grau variável entre os grupos.

A região rostral da lamela coanal do palatino (LC: Figs 5, $13,16,18)$ é pouco projetada dorsalmente, não alcançando a margem ventral do ectetmóide, exceto em Coraciidae e em Pelargopsis capensis e P. melanorhyncha (Daceloninae) em que é desenvolvida. A projeção dorsal da região caudal da lamela 
coanal é menor que na região rostral, exceto em Todidae (LC: Fig. 11) em que a lamela coanal é totalmente reduzida. Em Bucerotidae a lamela coanal do palatino está indistinta.

O processo pterigóideo do palatino mostra-se curto em Alcedininae Cerylinae (Ptp: Fig. 21), (Ptp: Fig. 27), Coraciidae, Leptosomidae e Upupidae, e longo em Meropidae (Ptp: Fig. 20), Todidae (Ptp: Fig. 26), Phoeniculidae, Bucerotidae e na maioria dos Daceloninae, exceto em Pelargopsis capensis e P. melanorhyncha em que é menor. Em Momotidae há variações (Pascotto \& DONATELLi 2003).

O ângulo rostromedial é reduzido, exceto em Momotidae, Todidae (ar: Fig. 26), Upupidae e Phoeniculidae em que apresenta uma discreta projeção rostral. Entretanto, em Momotidae e Todidae o ângulo rostromedial difere de Phoeniculidae e Upupidae por não se articular ou contatar com o maxilopalatino. Em Bucerotidae as porções direita e esquerda encontram-se fundidas entre si por uma considerável extensão e rostromedialmente com a porção caudomediana do maxilopalatino, ocorrendo a oclusão da face rostroventral da fossa coanal.

O processo transpalatino está presente em Alcedininae (pT: Figs 7, 27), Cerylinae (pT: Figs 16, 21), Daceloninae (pT: Fig. 17), Phoeniculidae e Upupidae, estando ausente nas demais famílias. Nos grupos em que o processo transpalatino inexiste, o ângulo caudolateral (ac: Fig. 26) é arredondado, exceto em Bucerotidae em que ele está indistinto. Na maioria das espécies de Meropidae (ac: Fig. 20) verifica-se uma discreta projeção caudal, exceto em Merops variegatus em que ele é amplamente arredondado; em Nyctyornis athertoni e M. bulocki ocorre variação individual.

Em Meropidae (V: Fig. 20) e Coraciidae o vômer é longo, enquanto que em Daceloninae ele é vestigial, laminar, achatado lateromedialmente e disposto no sentido dorsoventral em Dacelo novaeguineae, D. leachii, D. gaudichaud, Pelargopsis capensis e P. melanorhyncha, estando praticamente ausente nas demais espécies. Nos calaus (Bucerotidae) encontra-se variação específica, sendo laminar, mas vestigial na maioria das espécies. Em Bucorvus abyssinicus, B. cafer, e Aceros cassidix (Temminck, 1823) verifica-se desenvolvimento intermediário, enquanto que o maior desenvolvimento do vômer é observado em Ceratogymna elata, na qual ele se contata com o maxilopalatino. Em Tockus hartlaubi Gould, 1861 e Buceros vigil ele está ausente. Nos demais grupos o vômer está ausente.

O pterigóide é reto, exceto em Todidae (Pt: Fig. 11) e em algumas espécies de Alcedininae, como em Ceyx erithacus, $C$. lepidus, C. fallax, C. melanurus, Ispidina lecontei e I. picta em que ele possui uma pequena curvatura dorsomedial. A extremidade rostral do pterigóide apresenta-se reduzida em Meropidae (Pt: Fig. 20), Todidae (Pt: Fig. 26), Alcedininae (Pt: Fig. 27), Daceloninae, Coraciidae, Leptosomidae e Bucerotidae; em Brachypteraciidae, Phoeniculidae e Upupidae é pouco expandida lateromedialmente. Em Momotidae (Pascotto \& Donatelli 2003) verifica-se variação específica. Em Cerylinae ela também é reduzida, mas em Megaceryle lugubris (cpPt: Fig. 21), M. alcyon,
Chloroceryle amazona, C. americana, C. inda e C. aenea há a formação de uma conspícua crista que amplia sua área, estando ausente nas demais espécies. Em Ceryle rudis ocorre variação individual, podendo estar presente em alguns espécimes.

A porção rostral do arco jugal é expandida lateromedialmente e achatada dorsoventralmente em Cerylinae (exJA: Figs 4, 21) e nas espécies de Pelargopsis Gloger, 1841 (Daceloninae), mostrando desenvolvimento menor nos demais grupos, nos quais restringe-se rostralmente à área de contato entre jugal e lacrimal. Em Bucerotidae tal porção é reduzida, não havendo expansão lateromedial. Já a extremidade caudal do arco jugal é reta em Coraciidae (J: Fig. 12), Brachypteraciidae (J: Figs 13, 19), Alcedininae (J: Fig. 15), Cerylinae (J: Fig. 16), Daceloninae (J: Fig. 17), Leptosomidae e Bucerotidae, enquanto que em Phoeniculidae (J: Fig. 5), Todidae (J: Fig. 11), Meropidae (J: Fig. 18), Momotidae e Upupidae tal extremidade apresenta curvatura dorsal.

O processo orbital do quadrado é longo e ultrapassa medialmente a área muscular áspera em Daceloninae (OrpQ: Fig. 17), Todidae, Momotidae, Coraciidae, Brachypteraciidae, Leptosomidae, Phoeniculidae e Upupidae. Este processo tem desenvolvimento menor e restringe-se à área muscular áspera em Cerylinae e Meropidae. Em Alcedininae (OrpQ: Fig. 15) ele possui a base ampla mas é curto, não alcançando a área muscular áspera, e a margem dorsal diminui abruptamente em direção ventral. Em Bucerotidae encontra-se variação específica, sendo longo na maioria das espécies, exceto nas espécies de Tockus, Ocyceros Hume, 1873, Penelopides Reichenbach, 1849 e em Anorrhinus galeritus em que é menor e restringe-se à área muscular áspera, terminando antes ou na sua região mediana.

O côndilo caudal do processo mandibular do quadrado encontra-se fundido lateroventralmente com o côndilo lateral em Phoeniculidae (clQ+ccQ: Fig. 5), Upupidae e Leptosomidae, sendo distintos nos demais grupos (ccQ, clQ: Figs 12, 14, 17, 20, 27). Em Cerylinae ocorre variação específica (РАsсотTo et al. 2006).

O côndilo medial do processo mandibular do quadrado é o maior dos três côndilos em Cerylinae (cmQ: Figs 14, 21), Bucerotidae (cmQ: Fig. 22), Alcedininae (cmQ: Fig. 27), Daceloninae, Phoeniculidae e Upupidae, sendo nos demais grupos menor que o côndilo lateral. Em Leptosomidae torna-se difícil tal comparação, uma vez que o côndilo medial é estreito e o côndilo lateral mais arredondado. Exceto em Meropidae, o côndilo medial é o que tem maior projeção ventral dentre os Coraciiformes (cmQ: Figs 14, 22).

\section{Ossa Mandibulae}

A região sinfisiária abrange cerca de $50 \%$ ou metade do comprimento total da mandíbula em Alcedininae, Cerylinae, Meropidae, Phoeniculidae (Si: Fig. 28), Upupidae e Bucerotidae; cerca de 30\% ou 1/3 em Todidae (Si: Fig. 29) e Brachypteraciidae; e com desenvolvimento menor em Leptosomidae, ocupando cerca de $15 \%$ do desenvolvimento da mandíbula. Em Daceloninae ocorre variação específica, pois ocupa cerca de $50 \%$ do 
comprimento total da mandíbula na maioria das espécies, exceto em Halcyon chelicuti (Stanley, 1814) e Todiramphus winchelli em que ocupa cerca de $30 \%$. Para as variações de Momotidae, vide Pascotto \& Donatelli (2003).

O ângulo mandibular é reduzido, o que confere à mandíbula uma discreta curvatura ventral em Meropidae e na maioria das espécies de Bucerotidae, exceto no espécime macho de Buceros bicornis e em Tockus deckeni e Aceros comatus nos quais é mais acentuada, e em Buceros vigil (Fig. 30) em que ela é praticamente reta. Em Alcedininae, Cerylinae (Fig. 31), Todidae, Coraciidae e Leptosomidae a mandíbula é praticamente reta. Dentre os Brachypteraciidae, evidencia-se uma leve curvatura ventral em Brachypteracias leptosomus, enquanto que em Atelornis pittoides e A. crossleyi ela é pouco mais acentuada. O mesmo é encontrado nos Phoeniculidae, sendo a curvatura ventral da mandíbula acentuada nas espécies de Phoeniculus (Fig. 33) - padrão este semelhante ao de Upupidae - e extremamente acentuada nas espécies de Rhinopomastus (Fig. 34). Nos martins-pescadores da subfamília Daceloninae, verifica-se uma curvatura dorsal conspícua em Dacelo novaeguineae (Fig. 32), D. leachii, D. gaudichaud e Melidora macrorrhina, sendo discreta nas demais espécies.

A fossa medial da mandíbula é extremamente reduzida, rasa e em forma de fenda em Todidae, Leptosomidae, Upupidae e Bucerotidae; em Phoeniculidae também se apresenta extremamente reduzida, mas mostra-se arredondada. Ela é ampla e curta em extensão em Brachypteraciidae, Alcedininae e Daceloninae, mas em Cerylinae ocorre variação específica. Nos Coraciidae a fossa medial é pequena mas em forma de fenda, exceto em Eurystomus gularis em que tem desenvolvimento menor e é mais ovalada. Já nas espécies de Merops (Meropidae), ela é extremamente reduzida, estreita e curta, enquanto que nas espécies de Nyctyornis ela é um pouco mais longa, possuindo a forma de uma fenda. Em Momotidae (Pascotto \& Donatelli 2003) também há variação específica.

A fossa lateral da mandíbula (flM: Figs 31-34) é única, ampla e longa, sendo mais conspícua na porção rostral, exceto em Momotidae (Pascotto \& Donatelli 2003). Em Bucerotidae (flM: Fig. 30) ela é plana e em Coraciidae há variação específi$\mathrm{ca}$, sendo arredondada, extremamente reduzida e restrita à porção rostral da região intermediária nas espécies de Eurystomus, diferentemente das espécies de Coracias em que a fossa lateral é longa, estendendo-se por quase toda a região intermediária.

O processo coronóide 1 (pc 1: Figs 31, 32) e o processo coronóide 2 são reduzidos, mas distintos entre si, exceto em Bucerotidae em que se apresentam fundidos, formando uma conspícua crista óssea que se destaca da borda dorsal na região caudal da mandíbula.

O processo medial da mandíbula é longo, estreito e espesso, especialmente na sua base em Meropidae, Todidae (pmM: Fig. 29), Coraciidae, Momotidae, Leptosomidae e Brachypteraciidae, enquanto que em Alcedininae, Cerylinae, Phoeniculidae (pmM: Fig. 28), Upupidae e Bucerotidae apresenta-se curto, amplo e espesso. Em Daceloninae ocorre variação específica, sendo curto, amplo e espesso na maioria das espécies, exceto nas espécies de Pelargopsis em que é ainda mais curto.

O forame pneumático abre-se quase na extremidade distal do processo medial em Alcedininae, Cerylinae, Daceloninae, Phoeniculidae (fp: Fig. 28), Upupidae e Bucerotidae. Em Momotidae, Todidae (fp: Fig. 29), Meropidae, Leptosomidae, Coraciidae e Brachypteraciidae abre-se na região dorsomediana do processo medial da mandíbula.

O típico processo retroarticular da mandíbula está presente apenas em Phoeniculidae (pr: Figs 28, 33-35), Upupidae e Bucerotidae (pr: Fig. 30). Em Todidae (dcM: Fig. 29) e Coraciidae nota-se uma projeção caudal arredondada e espessa na porção dorsocaudal da mandíbula - região caudal à fossa articular quadrática - sendo que em Todidae (dcM: Fig. 29) tal região parece ser maior que nos demais grupos devido à extrema redução óssea da fossa articular quadrática, mas nesses dois grupos não há formação do processo retroarticular.

A fossa articular quadrática é profunda em virtude do extremo desenvolvimento da crista transversa em Daceloninae, Coraciidae, Leptosomidae, Phoeniculidae (faQ: Fig. 28), Upupidae e Bucerotidae, e rasa em Alcedininae, Momotidae, Meropidae e Brachypteraciidae. Em Todidae (Fig. 29) é extremamente reduzida ou praticamente ausente, enquanto que em Cerylinae ocorre variação específica (РАscotTo et al. 2006). O tubérculo intercotilar apresenta-se extremamente desenvolvido em Momotidae e Meropidae; desenvolvido em Alcedininae, Daceloninae, Todidae, Coraciidae e Leptosomidae; reduzido em Cerylinae, Phoeniculidae e Upupidae; e rudimentar em Brachypteraciidae e Bucerotidae.

As cótilas caudal e lateral da mandíbula, na borda dorsolateral da fossa articular quadrática, são indistintas devido à ausência do processo intercondilar separando-as em Alcedininae, Momotidae, Meropidae, Todidae (col+coc: Fig. 29), Coraciidae, Leptosomidae, Brachypteraciidae, Phoeniculidae (col+coc: Figs 28, 34) e Upupidae. Já em Cerylinae, Daceloninae e Bucerotidae as cótilas lateral (col: Figs 30-32) e caudal (coc: Figs 30-32) são distintas e divididas pelo processo intercondilar (pi: Figs 30-32). A porção rostral da cótila lateral evidencia o processo rostrolateral (prl: Figs 28, 31-33), destacando-se do ramo da mandíbula, com exceção de Leptosomidae e Bucerotidae em que está ausente. Já o processo rostromedial mostra-se inconspícuo, exceto em Cerylinae, Daceloninae e Leptosomidae em que é conspícuo; em Bucerotidae está ausente.

A fossa caudal da mandíbula é rasa e longa lateromedialmente em todos os Coraciiformes, sendo ampla dorsoventralmente, especialmente devido ao desenvolvimento da crista transversa, em Daceloninae, Leptosomidae, Phoeniculidae (fcM: Fig. 35), Upupidae e Bucerotidae; mostra-se estreita em Alcedininae, Momotidae, Meropidae e Brachypteraciidae; e extremamente estreita em Todidae. Em Cerylinae encontra-se variação específica (РАsсотто et al. 2006), assim como em Coraciidae, em que ela é pouco mais ampla dorsoventralmente nas espécies de Coracias que de Eurystomus. 


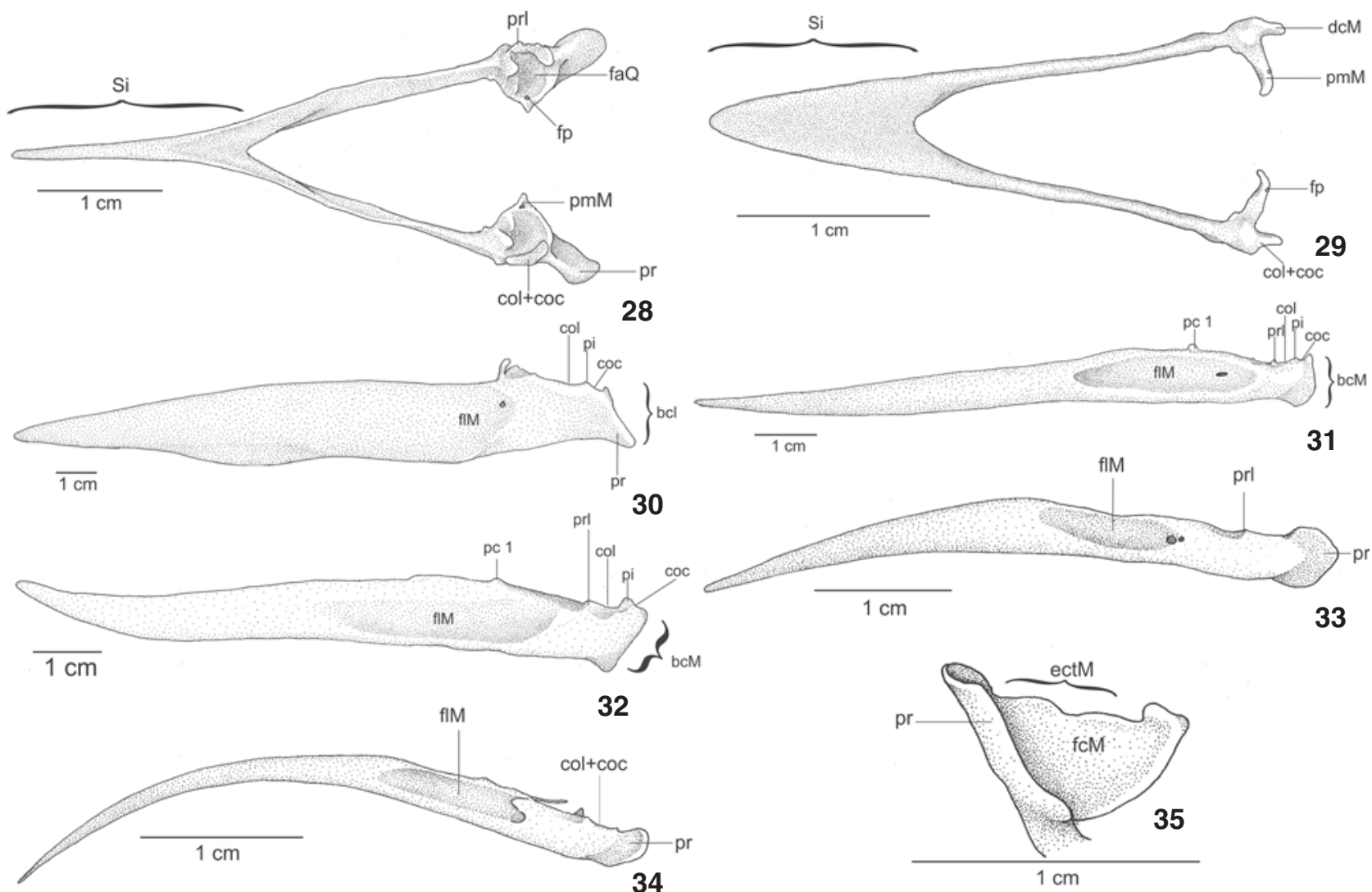

Figuras 28-35. Mandíbulas de: (28) Phoeniculus purpureus USNM 320948, vista dorsal; (29) Todus subulatus USNM 555798, vista dorsal; (30-34) vista lateral do ramo mandibular esquerdo de: (30) Buceros vigil USNM 18756; (31) Megaceryle torquata USNM 500429; (32) Dacelo novaeguineae MHNT 126; (33) Phoeniculus purpureus USNM 320948; (34) Rhinopomastus cyanomelas USNM 431715; (35) Phoeniculus purpureus USNM 320948, vista caudal do ramo mandibular esquerdo. (bcM) Borda caudolateral da mandíbula, (coc) cótila caudal da mandíbula, (col) cótila lateral da mandíbula, (col+coc) fusão das cótilas lateral e caudal da mandíbula, (dcM) região dorsocaudal da mandíbula, (ectM) emarginação da crista transversa da mandíbula, (faQ) fossa articular quadrática, (fcM) fossa caudal da mandíbula, (fIM) fossa lateral da mandíbula, (fp) forame pneumático, (pc 1) processo coronóide 1, (pi) processo intercondilar, (pmM) processo medial da mandíbula, (pr) processo retroarticular, (prl) processo rostrolateral da cótila lateral, (Si) região sinfisiária.

A margem dorsal da crista transversa é praticamente plana, não havendo emarginação em Coraciidae, Leptosomidae, Phoeniculidae (ectM: Fig. 35), Upupidae e Bucerotidae; profunda e ampla em Alcedininae, Momotidae e Brachypteraciidae e rasa e ampla em Meropidae, exceto em Merops ornatus em que possui amplitude menor. É extremamente profunda e ampla em Todidae, especialmente em virtude da ausência da crista transversa, enquanto que em Daceloninae ela tem a forma de semi-esfera, sendo profunda e pouco ampla. Em Cerylinae ocorre variação específica (РАsсотTO et al. 2006).

Em vista lateral, a borda caudolateral da mandíbula apresenta-se oblíqua, disposta dorsoventrorrostralmente em Meropidae, Todidae, Momotidae, Coraciidae, Leptosomidae e Brachypteraciidae; é arredondada em Alcedininae e Cerylinae (bcM: Fig. 31). Na maioria das espécies de Daceloninae, a borda caudo- lateral da mandíbula é oblíqua (bcM: Fig. 32), exceto nas espécies de Pelargopsis em que é arredondada e em Halcyon pileata em que ela é truncada, perpendicular ao ramo da mandíbula. Dentre os Bucerotidae ocorre variação, sendo oblíqua nos Bucerotinae (Fig. 30) e reta ou truncada nos Bucorvinae. Já em Upupidae e Phoeniculidae (Figs 33, 34) ela também é arredondada, mas devido à presença do processo retroarticular.

\section{DISCUSSÃO}

\section{Aspectos morfológicos}

Após a análise e comparação das estruturas osteológicas cranianas dos Coraciiformes, podem ser destacadas algumas características comuns à ordem Coraciiformes: presença da fossa lateroesfenóide, ausência do processo suprameático e ausência 
do típico processo retroarticular da mandíbula (presente apenas em Upupidae, Phoeniculidae e Bucerotidae), embora tais caracteres não sejam exclusivos dos Coraciiformes. O diagnóstico da ordem pela ausência de alguns caracteres também foi encontrado em dados miológicos por MAURER \& RAIKOW (1981), os quais reuniram os Coraciiformes pela ausência de dois músculos, o M. ambiens e o M. iliofemoralis externus, também ausentes em grupos próximos, como em Piciformes e em muitos Passeriformes.

A ausência do processo suprameático nos Coraciiformes não é exclusividade da ordem, uma vez que os Trogonidae (Trogoniformes), Galbulidae (Donatelli 1992), Capitonidae (Piciformes) e Dendrocolaptidae (Donatelu 1997) também não o possuem. Entretanto, difere de outros grupos de aves em que o processo suprameático mostra-se pouco desenvolvido, como em Crotophaginae (Posso \& Donatelu 2001) e Ramphastidae ou extremamente desenvolvido, como em Psophiidae (MARCEliano et al. 1997) e Picidae (Donatell 1996).

Nos Coraciiformes podem ser observados três estágios de desenvolvimento da fossa temporal. O maior desenvolvimento extensão e profundidade - é encontrado nos martins-pescadores (Alcedinidae), sendo que neles as fossas temporais direita e esquerda encontram-se dorsalmente na linha mediana do crânio, salvo raras exceções em que estão muito próximas. O menor desenvolvimento - extensão e profundidade - pode ser observado em Todidae, Leptosomidae, Upupidae e Phoeniculidae, sendo que nestes grupos a fossa temporal é praticamente plana. Entretanto, o menor desenvolvimento da fossa temporal dos Coraciiformes ainda é maior que em Trogonidae, os quais possuem a fossa temporal extremamente reduzida e plana, adquirindo a forma de uma semi-esfera. Já em Momotidae, Meropidae, Coraciidae, Brachypteraciidae e Bucerotidae a fossa temporal mostra desenvolvimento intermediário, sendo sua porção lateral mais profunda que a porção dorsal, semelhante ao padrão encontrado nos Piciformes. Analisando o trabalho de BurTon (1984), pode-se constatar ainda que o M. adductor mandibulae externus rostralis temporalis apresenta-se extremamente desenvolvido ou volumoso nos martins-pescadores (Alcedininae, Cerylinae e Daceloninae), reduzido em Todidae, Leptosomidae, Upupidae e Phoeniculidae, e com desenvolvimento intermediário em Momotidae, Meropidae, Coraciidae, Brachypteraciidae e Bucerotidae, corroborando a inferência de Donatelu (1992). Ainda, pode-se ir além e dizer que tanto o desenvolvimento da fossa temporal quanto o desenvolvimento e a conformação da fossa lateroesfenóide estão intimamente relacionados ao desenvolvimento e complexidade estrutural do $M$. adductor mandibulae externus rostralis temporalis.

Apesar da forma e do tamanho do bico - formado pela maxila superior e mandíbula - serem uma característica plástica na evolução das aves, muitos grupos podem ser diagnosticados simplesmente pela forma do bico, como os Psittacidae, Pelecanidae, Phoenicopteridae, Fringillidae, entre outros. Entretanto, a diferenciação entre espécies e gêneros com base apenas na forma e tamanho do bico nem sempre é possível, sendo que na maioria das vezes as diferenças são mínimas e podem ser apenas reflexos de diferentes preferências alimentares. Inferências evolutivas a respeito desse caráter somente podem ser feitas após uma análise cladística bem fundamentada. Dentre os Coraciiformes há uma variedade relativa de tamanhos e formas de bicos, os quais estão relacionados às diferentes táticas de forrageamento e alimentação. Entretanto, a diferenciação marcante é observada principalmente entre as famílias e, com raras exceções, em nível genérico e específico. Os martinspescadores (Alcedinidae) possuem diferenças marcantes de acordo com suas táticas de forrageamento: os martins-pescadores verdadeiramente piscívoros (Cerylinae) possuem um bico em forma lanceolada, ou seja, com a base caudal mais larga e evidenciando um estreitamento gradativo até a extremidade rostral extremamente afilada, sendo ainda tanto a maxila superior como a mandíbula praticamente retilíneas, não evidenciando curvaturas ventral ou dorsal. Essa forma lanceolada do bico dos Cerylinae permite movimentos mais eficazes embaixo d'água (BurTon 1984). Os Daceloninae possuem, na maioria das espécies, um bico relativamente curto, robusto e amplo na base, adequado para o esmagamento de insetos, sendo os mais amplos e robustos encontrados nos gêneros Dacelo Leach, 1815 e Melidora Lesson, 1830. Uma exceção marcante dessa subfamília é observada nas espécies do gênero Pelargopsis, que possuem o bico estreito e reto, muito semelhante à condição encontrada nos Cerylinae. A forma do bico de Pelargopsis também é uma adaptação em virtude ao modo de forrageamento dessas espécies, que regularmente se alimentam de peixes capturados por meio de mergulhos na água. Já os Alcedininae apresentam formas de bico intermediárias, especializadas tanto para a captura de peixes (especialmente as espécies do gênero Alcedo Linnaeus, 1758) quanto para o esmagamento de insetos. Entretanto, nenhuma espécie de Alcedininae possui formas cranianas características dos Cerylinae e/ou Daceloninae. O bico achatado e delgado dos Todidae é uma especialização para capturar insetos na parte inferior das folhas por meio de movimentos rápidos. Os Meropidae possuem um bico longo, relativamente estreito - exceto na base - e curvo ventralmente, refletindo sua tática de forrageamento que consiste na captura de insetos em vôo. Como a maioria das presas dos Meropidae (abelharucos) é composta basicamente de insetos venenosos, como abelhas e vespas, possivelmente o bico longo permite a essas aves imobilizarem suas presas e destruir os seus ferrões a uma certa distância do corpo antes de ingerí-las, a fim de evitarem receber ferroadas das próprias presas. Dentre os Meropidae, Nyctyornis possui o bico maior e mais robusto que as espécies de Merops e Meropogon, refletindo o modo de forrageamento freqüentemente em poleiros. Nos Coraciidae, tanto a maxila superior quanto a mandíbula são mais curtas e amplas em Eurystomus que em Coracias, refletindo assim as diferentes táticas de forrageamento dos representantes desses dois gêneros: o bico mais amplo das espécies do gênero Eurystomus pode favorecer o esmagamento de insetos capturados durante o vôo, enquanto que o bico mais 
estreito e longo de Coracias provavelmente facilite a captura de insetos no solo. Leptosomidae possui o bico mais amplo e espesso dentre os Coraciiformes, facilitando assim a captura e ingestão de presas relativamente grandes, tais como camaleões, lagartos, gafanhotos e outros insetos de grande porte (ForsHAW 1998, Goodman 2001), em relação ao tamanho corpóreo da ave. O bico amplo e robusto auxilia no esmagamento das presas, bem como tem maior resistência durante os movimentos de batida das presas contra um substrato para imobilizá-las antes de ingerí-las. Brachypteraciidae possui o bico muito semelhante ao dos Coraciidae, particularmente às espécies de Coracias, sendo o bico robusto e amplo especializado para a alimentação basicamente insetívora. Os Upupidae utilizam o bico longo e fino para revirar o solo à procura principalmente de insetos (Forshaw 1998, KRISTIN 2001), os quais são capturados e seguros inicialmente pela ponta do bico, sendo conduzidos para a parte posterior da boca por meio do levantamento da cabeça. Semelhante aos pica-paus (Picidae), os Phoeniculidae utilizam seu bico estreito, decurvo e longo para capturar, em substratos verticais, insetos e larvas escondidas nos troncos e galhos das árvores (BurTon 1984, Forshaw 1998). Certamente, o bico mais longo e decurvo de Rhinopomastus é uma adaptação ao forrageamento em flores de acácias, os quais sugam, principalmente, formigas que entram nas flores por meio de pequenas aberturas na corola. Já os calaus (Bucerotidae) apresentam um bico característico e único dentre as Aves. A forma estreita e alta do bico dos calaus é extremamente adequada à alimentação e limpeza do ninho por meio de uma fenda durante o período de clausura da fêmea (Kemp 1995, 2001). Contudo, é importante lembrar que a ranfoteca exerce um papel adicional e fundamental durante os processos de alimentação e outros comportamentos que envolvam o uso do bico, alterando assim sua forma e seu desenvolvimento (tamanho). Vale ressaltar que as características anatômicas aqui apresentadas são baseadas apenas no desenvolvimento e forma do bico ósseo - sem a ranfoteca, uma vez que a mesma nem sempre está preservada nos espécimes das coleções científicas.

Segundo Donatelli (1996), o processo esquamosal proporciona uma grande área de superfície para origem das aponeuroses e fibras musculares dos músculos adutores mandibulares externos. Entretanto, o processo esquamosal assume diferentes tamanhos nos Coraciiformes. É extremamente reduzido em Leptosomidae e Phoeniculidae, praticamente não evidenciando projeções, semelhante ao encontrado nos Galbulidae (Donatelli 1992). Conseqüentemente, não há área para inserção desse sistema muscular, estando o M. adductor mandibulae externus rostralis lateralis ausente em Leptosomidae e pouco desenvolvido em Phoeniculidae (BurTon 1984). Nos Cerylinae e Meropidae o processo esquamosal é reduzido, ocupando cerca de $1 / 4$ do desenvolvimento desde sua base no esquamosal até o arco jugal, seguido por Upupidae, com aproximadamente $1 / 3$ desse desenvolvimento, enquanto que nos demais grupos há variações específicas no desenvolvimento do processo esquamosal. Entretanto, o maior desenvolvimento do processo esquamosal encontrado nos Coraciiformes não ultrapassa cerca de metade da distância desde o esquamosal até o arco jugal. Isso significa que podemos encontrar uma certa complexidade e diversidade nos feixes musculares do sistema adutor mandibular externo dos Coraciiformes, uma vez que o $M$. adductor mandibulae externus foi relatado por BURTON (1984) como o sistema muscular mais complexo e com maior variação estrutural nos Coraciiformes e Piciformes. Um estudo minucioso do sistema muscular craniano dos Coraciiformes pode ser encontrado em BurTon (1984).

Dentre os grupos em que o típico lacrimal não ocorre, ele está completamente ausente em Momotidae (РАsсотто \& Donatelli 2003) e, nos indivíduos adultos de Bucerotidae, ele se funde com o ectetmóide formando o complexo lacrimalectetmóide. Phoeniculidae e Upupidae possuem o mesmo padrão encontrado nos calaus (Bucerotidae) e, possivelmente, também ocorre fusão entre lacrimal e ectetmóide. Porém, faz-se necessário o estudo de espécimes jovens para a ratificação de tal hipótese. O extremo desenvolvimento do processo supra-orbital do lacrimal nos Coraciiformes é observado em Daceloninae (Dacelo), sendo que tal desenvolvimento parece estar relacionado à maior proteção da região orbital durante os movimentos da cinese craniana formando, assim, uma superfície maior para inserção da membrana periorbital (CRACRAFT 1968).

O processo orbital do quadrado é o local de origem do $M$. pseudotemporalis profundus (BOCK 1964, BÜHLER 1981). BURTON (1984) descreveu o processo orbital do quadrado como sendo estreito ou vestigial em Cerylinae e Alcedininae. Entretanto, o termo vestigial não é apropriado para os Alcedinidae, uma vez que nesses martins-pescadores o processo orbital do quadrado é curto, mas não vestigial, tampouco para os Cerylinae. A redução do processo orbital do quadrado nos Alcedininae, provavelmente, não está relacionada à ausência do $M$. pseudotemporalis profundus, uma vez que BurTon (1984) também constatou a ausência de tal músculo em algumas espécies de Cerylinae.

\section{Aspectos anátomo-funcionais}

É importante discutir a osteologia craniana considerando-se o crânio como uma unidade funcional, na qual estão envolvidos os sistemas muscular e aponeurotendinoso. Dada a grande diversidade estrutural encontrada no crânio dos Coraciiformes, supõe-se que haja uma grande complexidade funcional dos ossos e estruturas associadas ao crânio dessas aves. Entretanto, inferências a respeito da anatomia funcional dos Coraciiformes tornam-se restritivas, especialmente por não incluir séries de crânios de espécimes em diferentes estágios de desenvolvimento ontogenético, o que é raro em coleções científicas.

Segundo Bоск (1964), todas as aves possuem cinese craniana - na qual a maxila superior realiza movimentos total ou parcialmente independentes em relação à caixa craniana - sendo a condição básica ou primitiva das aves a procinese, a qual envolve articulação entre os ossos frontal e nasal por meio da zona flexória craniofacial. No crânio procinético, a maxila superior é 
uma unidade óssea inflexível, sendo a região de articulação a zona flexória craniofacial (Bock 1966, BÜHLER 1981, Zusi 1984), local mais frágil da maxila superior (Bоск 1966). A procinese é encontrada nos Coraciiformes, embora a zona flexória craniofacial não seja uma sutura verdadeira ou completa em alguns grupos, como em Coraciidae e Leptosomidae. Assim, a diferenciação da zona flexória craniofacial dos Coraciiformes provavelmente interfere na dimensão e liberdade dos movimentos durante a cinese craniana, podendo ser limitante em Coraciidae e Leptosomidae. O extremo desenvolvimento (amplitude) da zona flexória craniofacial é encontrada nos Alcedinidae, particularmente em Cerylinae, nos quais a zona flexória craniofacial é mais ampla rostrocaudalmente que nos demais grupos de Coraciiformes e em Trogoniformes, sendo que a presença de uma zona flexória craniofacial verdadeira é considerada rara dentre as aves (Zusi 1984).

Embora ainda haja discordâncias sobre os movimentos exatos do osso quadrado durante a abertura do bico, praticamente todos os autores concordam no que diz respeito à função desempenhada pelo quadrado durante a cinese craniana: ele é o ponto inicial a partir do qual se originam todos os movimentos cinéticos cranianos (Bock 1964, BüHLER 1981, Zusi 1981, BurToN 1984). O arco jugal, associado ao processo orbital do lacrimal, exerce importante papel na estabilização do quadrado durante a cinese craniana, formando um perfeito encaixe: a face ventral da extremidade distal do processo orbital do lacrimal pode apresentar, em alguns grupos, um sulco ou uma depressão longitudinal na qual se encaixa o arco jugal, que direciona os movimentos do quadrado (CRACRAfT 1968). Entretanto, nos martins-pescadores verdadeiramente piscívoros (Cerylinae) e em Pelargopsis capensis (Daceloninae) ocorre exatamente o oposto: o arco jugal é que apresenta uma concavidade ou depressão longitudinal, na qual provavelmente desliza a porção distal do processo orbital do lacrimal, que tem uma forma bulbosa, durante os movimentos da cinese craniana. Nos demais Coraciiformes nenhuma das duas condições acima descritas são encontradas.

Durante as atividades diárias que envolvem o uso do bico, as aves estão sujeitas a uma grande variedade de forças externas atuando durante a cinese craniana. Durante o esmagamento de alimentos, por exemplo, em que são realizados movimentos ascendentes, descendentes e laterais do bico, as aves podem se deparar com algumas partes mais duras das presas, necessitando de forças adicionais que muitas vezes poderiam causar rupturas de ligamentos ou de fibras musculares, ou ainda desarticulações. Essas forças adicionais requerem maior esforço muscular, o que talvez explicaria a evolução de mecanismos adicionais que atuariam na prevenção de rupturas e na quebra ou desarticulações, tanto da zona flexória craniofacial quanto do sistema palatinopterigóide-quadrado (Cracraft 1968). Entretanto, como explicar a existência de alguns pontos ósseos adicionais em apenas alguns grupos de aves? Segundo o mesmo autor, a incapacidade ou deficiência muscular em responder às forças extras poderia aumentar os riscos de rupturas e desarticulações favorecendo, assim, a seleção de estruturas ou pontos de apoios adicionais em determinadas espécies ou grupos.

$\mathrm{O}$ processo orbital do quadrado parece funcionar como um dispositivo de segurança na cessação da protração da maxila superior, ou seja, evita sua elevação excessiva. Isso se dá quando o processo orbital do quadrado contacta a parede caudal da órbita - região lateroesfenóide. Entretanto, alguns grupos de Aves possuem uma intumescência na região lateroesfenóide, a área muscular áspera, responsável pela antecipação do contado entre a região lateroesfenóide e o processo orbital do quadrado (BURTON 1984). Nos Coraciiformes a área muscular áspera é desenvolvida na maioria das famílias, exceto em Leptosomidae, Meropidae e Phoeniculidae em que se mostra praticamente ausente ou plana, e em Todidae em que apenas sua porção ventral é ossificada. De acordo com as suposições de BurTon (1984), seria de se esperar que nos grupos em que o processo orbital do quadrado estivesse reduzido ocorresse um desenvolvimento acentuado da área muscular áspera, ou que nos grupos em que ela estivesse reduzida ou ausente houvesse um desenvolvimento acentuado do processo orbital do quadrado. No entanto, essa relação não é encontrada nos Coraciiformes: não ocorre desenvolvimento diferenciado ou excessivo em relação à área muscular áspera nos Alcedininae, tampouco desenvolvimento acentuado para o processo orbital do quadrado de Todidae, Meropidae, Leptosomidae, Phoeniculidae ou Bucerotidae. Assim, torna-se difícil associar o desenvolvimento da área muscular áspera ao desenvolvimento do processo orbital do quadrado e atribuir como função primária a prevenção da protração da maxila superior, sem mesmo nos certificar se realmente há contato entre essas duas estruturas nas aves in vivo.

O processo medial da mandíbula serve como área de inserção muscular: na sua porção caudal insere-se o $M$. depressor mandibulae, na base o M. pterygoideus dorsalis medialis e na porção rostral o M. pterygoideus ventralis medialis (BocK 1960, BuRTON 1984). O maior desenvolvimento do processo medial da mandíbula é encontrado na maioria dos Coraciiformes, como em Momotidae, Todidae, Meropidae, Coraciidae, Phoeniculidae e Brachypteraciidae, sendo nestes grupos longo e estreito, exceto na base ventral, enquanto que em Alcedininae, Cerylinae, Daceloninae, Upupidae e Bucerotidae mostra-se curto, sendo mais amplo que longo, exceto na extremidade distal. Воск (1960) relaciona o desenvolvimento e a forma do processo medial da mandíbula com o desenvolvimento do $M$. depressor mandibulae e do M. pterygoideus. Burton (1984) diagnosticou que o M. depressor mandibulae é uniforme e pouco desenvolvido nos Coraciiformes, exceto nos Upupidae e Phoeniculidae, não sendo possível, portanto, estabelecer qualquer relação entre o desenvolvimento do processo medial da mandíbula e do M. depressor mandibulae.

\section{CONCLUSÕES}

O estudo do esqueleto dos táxons da ordem Coraciiformes sensu WeTMORE (1960) constatou a diversidade morfológica existente entre eles, sendo difícil diagnosticá-la ou encontrar carac- 
teres que sejam comuns a todos os táxons. Porém, verificou-se que as famílias apresentam caracteres osteológicos particulares que as diferenciam, como também há caracteres que são comuns a dois ou mais táxons, especialmente nas categorias de gêneros, subfamílias e famílias, sendo indícios de que a osteologia pode ser uma fonte viável para a proposição de hipóteses filogenéticas para a ordem Coraciiformes (estudo em conclusão pelos autores). Apesar da diversidade morfológica, a formação do complexo lacrimal-ectetmóide e do processo retroarticular da mandíbula permite caracterizar os Upupidae, Phoeniculidae e Bucerotidae em um grupo à parte das demais famílias de Coraciiformes, mas somente um estudo filogenético é capaz de esclarecer tal hipótese de relacionamento.

\section{AGRADECIMENTOS}

ÀCoordenadoria de Aperfeiçoamento de Pessoal de Ensino Superior, à Pró-Reitoria de Pós-Graduação da Universidade de São Paulo, a Frank M. Chapman Memorial Fund (AMNH) e à Smithsonian Institution (Office of Fellowships and Grants) pela bolsa de estudos e pelos auxílios financeiros concedidos à M.C. Pascotto; ao Conselho Nacional de Desenvolvimento Científico e Tecnológico, pela Bolsa de Produtividade em Pesquisa de E. Höfling e a dois revisores anônimos. Ao American Museum of Natural History (AMNH), New York, EUA; à Coleção de Aves, Departamento de Zoologia, Universidade de São Paulo, São Paulo, Brasil (AZ); ao Field Museum of Natural History, Chicago, EUA (FMNH); à Louisiana State University Museum of Natural Science, Baton Rouge, EUA (LSUMNS); ao National Museum of Natural History, Smithsonian Institution, Washington, EUA (USNM); ao National Museum of Victoria, Australia (NMV); à University of Kansas Museum of Natural History, Lawrence, EUA (KUMNH); à University of Michigan Museum of Zoology, Ann Arbor, EUA (UMMZ); ao Museu de História Natural de Taubaté, Brasil (MHNT); e ao Museu Paraense Emílio Goeldi, Belém, Brasil (MPEG), tanto pelos empréstimos de material osteológico como pelas visitas de M.C. Pascotto a algumas coleções científicas.

\section{REFERÊNCIAS BIBLIOGRÁFICAS}

BaUmel, J.J. \& L.M. WitMer. 1993. Osteologia, p. 45-132. In: J.J. Baumel; A.S. King, J.E. Breazile; H.E. Evans \& J.C.V. Berge (Eds). 1993. Handbook of avian anatomy: Nomina Anatomica Avium. Cambridge, Nutall Ornithological Club, $2^{\text {nd }}$ ed., 793p.

Beddard, F.E. 1896. Contributions to the anatomy of Picarian birds. Part III. On some points in the anatomy of the kingfishers. Proceedings of the Zoological Society of London, London, 1896: 603-606.

Воск, W.J. 1960. Secondary articulation of the avian mandible. Auk, Washington, 77 (1): 19-55.

Воск, W.J. 1964. Kinetics of the avian skull. Journal of Morphology, Philadelphia, 114: 1-41.

Воск, W.J. 1966. An approach to the functional analysis of bill shape. Auk, Washington, 83: 10-51.
BÜHLER, P. 1981. Functional anatomy of the avian jaw apparatus, p. 439-468. In: A.S. King \& J. McLelland (Eds). Form and Functions in Birds. London, Academic Press, vol. 2, 496p.

Burton, P.J.K. 1978. The basisphenoid notch of kingfishers. Bulletin of British Ornithological Club, London, 98 (2): 68-74.

BURTON, P.J.K. 1984. Anatomy and evolution of the feeding apparatus in the avian orders Coraciiformes and Piciformes. Bulletin of the British Museum (Natural History), London, 47 (6): 331-443.

Cracraft, J. 1968. The lacrimal-ectethmoid bone complex in birds: a single character analysis. American Midland Naturalist, New York, 80 (2): 316-359.

CRACRAFT, J. 1971. The relationships and evolution of the rollers: families Coraciidae, Brachypteraciidae and Leptosomatidae. Auk, Washington, 88: 723-752.

Donatelli, R.J. 1992. Cranial osteology and myology of the jaw apparatus in the Galbulidae (Aves, Piciformes). Arquivos de Zoologia, São Paulo, 32 (1): 1-32.

Donatelli, R.J. 1996. The jaw apparatus of the Neotropical and of the Afrotropical woodpeckers (Aves: Piciformes). Arquivos de Zoologia, São Paulo, 33 (1): 1-70.

Donatelli, R.J. 1997. Osteologia e miologia cranianas de Dendrocolaptidae (Passeriformes, Tyranni) 1. Gêneros Glyphorhynchus, Campylorhamphus, Dendrocincla, Xiphorhynchus e Dendrocolaptes. Ararajuba, Belo Horizonte, 5 (1): 19-37.

FeducCiA, A. 1975a. Morphology of the bony stapes (Columella) in the Passeriformes and related groups: evolutionary implications. University of Kansas, Museum of Natural History Miscellaneous Publications, Kansas, 63: 1-34.

FeducCIA, A. 1975b. The bony stapes in the Upupidae and Phoeniculidae: evidence for commom ancestry. The Wilson Bulletin, Lawrence, 87 (3): 416-417.

Flausino Jr, O.A.; R.J. Donatelli \& M.C. Pascotto. 1999. Osteologia e miologia cranianas de Momotus momota (Coraciiformes, Momotidae). Ararajuba, Londrina, 7 (2): 109-124.

Forbes, W.A. 1880. On the anatomy of Leptosoma discolor. Proceedings of the Zoological Society of London, London, n. 1880: $465-474$.

FoRBES, W.A. 1882. On some points in the anatomy of the todies (Todidae), and on the affinities of that group. Proceedings of the Zoological Society of London, London, 1882: 442450.

ForshaW, J. 1998. Encyclopedia of Birds. San Diego, Academic Press, $2^{\text {nd }}$ ed., 240p.

FrY, C.H. 2001a. Family Meropidae (bee-eaters), p. 286-341. In: J. Del Hoyo; A.S. Elliot \& J. Sargatal (Eds). Handbook of the birds of the world. Barcelona, Lynx Edicions, vol. 6, 589p.

FrY, C.H. 2001b. Family Coraciidae (rollers), p. 342-376. In: J. del Hoyo; A.S. Elliot \& J. Sargatal (Eds). Handbook of the birds of the world. Barcelona, Lynx Edicions, vol. 6, 589p.

FrY, C. H.; K. FrY. 1999. Kingfishers, bee-eaters, and rollers. New Jersey, Princeton University Press, 324p. 
Garrod, A.H. 1875. On the disposition of the deep Plantar tendons in different birds. Proceedings of the Zoological Society of London, London, 1875: 339-348.

Garrod, A.H. 1878. On the systematic position of the Momotidae. Proceedings of the Zoological Society of London, London, 1878: 100-102.

Goodman, S.M. 2001. Family Leptosomidae (cuckoo-roller), p. 390-395. In: J. Del Hoyo; A.S. Elliot \& J. Sargatal (Eds). Handbook of the birds of the world. Barcelona, Lynx Edicions, vol. 6,589 p.

HöFling, E. \& J-P. GaSc. 1984. Biomécanique du crâne et du bec chez Ramphastos (Ramphastidae, Aves). I - Description des éléments anatomiques. Gegenbaurs Morph. Jahrb, Leipzig, 130 (1): 125-147.

JAMES, H.F. 2004. The osteology and phylogeny of the Hawaiian finch radiation (Fringillidae: Drepanidini), including extinct taxa. Zoological Journal of the Linnean Society, London, 141: 207-255.

JoluiE, M. 1957. The head skeleton of the chicken and remarks on the anatomy of this region in other birds. Journal of Morphology, Philadelphia, 100 (4): 389-436.

Kemp, A. 1995. The Hornbills. Bucerotiformes. New York, Oxford University Press, 302p.

KemP, A. 2001. Family Bucerotidae (hornbills), p. 130-249. In: J. del Hoyo; A.S. Elliot \& J. Sargatal (Eds). Handbook of the birds of the world. Barcelona, Lynx Edicions, vol. 6, 589p.

Kepler, A.K. 2001. Family Todidae (todies), p. 250-263. In: J. DEL Hoyo; A.S. Elliot \& J. Sargatal (Eds). Handbook of the birds of the world. Barcelona, Lynx Edicions, vol. 6, 589p.

Kristin, A. 2001. Family Upupidae (hoopoe), p. 396-411. In: J. del Hoyo; A.S. Elliot \& J. Sargatal (Eds). Handbook of the birds of the world. Barcelona, Lynx Edicions, vol. 6, 589p.

LANGRAND, O. 2001. Family Brachypteraciidae (ground-rollers), p. 378-388. In: J. del Hoyo; A.S. Elliot \& J. Sargatal (Eds). Handbook of the birds of the world. Barcelona, Lynx Edicions, vol. 6, 589p.

Ligon, J.D. 2001. Family Phoeniculidae (woodhoopoes), p. 412-434. In: J. Del Hoyo; A.S. Elliot \& J. SARGaTAL (Eds). Handbook of the birds of the world. Barcelona, Lynx Edicions, vol. 6, 589p.

LivezeY, B.C. \& R.L. Zusi. 2001. Higher-order phylogenetics of modern Aves based on comparative anatomy. Netherlands Journal of Zoology, Leiden, 51 (2): 179-205.

LOWE, P.R. 1948. What are the Coraciiformes? Ibis, London, 90: $572-582$.

Marceliano, M.L.V.; R.J. Donatelli, E. Höfling \& S.R. Posso. 1997. Osteologia e miologia cranianas de Psophiidae (Aves: Gruiformes). Boletim do Museu Paraense Emílio Goeldi, Série Zoologia, Belém, 13 (1): 39-76.

Maurer, D.R. \& R.J. Raikow. 1981. Appendicular myology, phylogeny, and classification of the avian order Coraciiformes (including Trogoniformes). Annals of Carnegie Museum, Pittsburgh, 50 (18): 417-434.

MorRIs, P. \& F. HAwKINs. 1998. Birds of Madagascar. A photo- graphic guide. New Haven, Yale University Press, 316p.

MurIE, J. 1872. On the skeleton of Todus, with remarks as its allies. Proceedings of the Zoological Society of London, London, 1: 664-680.

Pascotto, M.C. \& R.J. Donatelli. 2003. Cranial osteology in Momotidae (Aves: Coraciiformes). Journal of Morphology, Philadelphia, 258 (1): 32-48.

Pascotto, M.C.; E. Höfling \& R.J. Donatelli. 2006. The Ringed Kingfisher, Ceryle or Megaceryle torquata (Cerylinae, Alcedinidae, Coraciiformes)? An osteological view. Ornitología Neotropical, Quebec, 17 (4): in press.

Posso, S.R. \& R.J. Donatelli. 2001. Cranial osteology and systematic implications in Crotophaginae (Aves, Cuculidae). Journal of Zoology Systematic Evolutionary Research, Berlin, 39: 247-256.

Posso, S.R. \& R.J. DonateluI. 2006. Skull and mandible formation in the cuckoos (Aves, Cuculidae): contributions to the nomenclature in avian osteology and systematics. European Journal Of Morphology, Londres, 43: in press.

SClater, P.L. 1865. On the structure of Leptosoma discolor. Proceedings of the Zoological Society of London, London, 1865: 682-689.

Sеввонм, H. 1890. An attempt to diagnose the subclass Coraciiformes and the orders, suborders, and families comprised therein. Ibis, London, 32: 200-205.

Sibley, C.G. \& B.L. Monroe. 1990. Distribution and taxonomy of birds of the world. New Halen, Yale University Press, 976p.

Snow, D.W. 2001. Family Momotidae (motmots), p. 250-263. In: J. Del Hoyo; A.S. Elliot \& J. Sargatal (Eds). Handbook of the birds of the world. Barcelona, Lynx Edicions, vol. 6, 589p.

STRICKLAND, H.E. 1843. On the structure and affinities of Upupa, Lin., and Irrisor, Lesson. Annals of Magazine of Natural History, London, 12 (1): 238-243.

Verheyen, R. 1955. Analyse du potentiel morphologique et considérations sur la systématique des Coraciiformes (Wetmore 1934). Buletin of the Institut Royal des Sciences Naturelles de Belgique, Bruxelles, 31 (93): 1-19.

Wetmore, A. 1960. A classification for the birds of the world. Smithsonian Miscellaneous Collections, Washington, 139 (11): 1-37.

Woodall, P.F. 2001. Family Alcedinidae (kingfishers), p. 130-249. In: J. Del Hoyo; A.S. Elliot \& J. Sargatal (Eds). Handbook of the birds of the world. Barcelona, Lynx Edicions, vol. 6, 589p.

ZusI, R.L. 1978. The interorbital septum in cardueline finches. Bulletin of the British Ornithological Club, London, 98: 5-10.

Zusi, R.L. 1981. Patterns of diversity in the avian skull, p. 391437. In: A. S. King \& J. McLelland (Eds). Form and Function in Birds. London, Academic Press, vol. 1, 566p.

Zusi, R.L. 1984. Myology of the Purple-throated Carib (Eulampis jugularis) and other hummingbirds (Aves, Trochilidae). Smithsonian Contributions to Zoology, Washington, 385: 1-70. 
Apêndice. Os espécimes utilizados pertencem às coleções científicas dos museus: American Museum of Natural History, New York, USA (AMNH); Coleção de Aves do Departamento de Zoologia da Universidade de São Paulo, São Paulo, Brasil (AZ); Field Museum of Natural History, Chicago, IL, USA (FMNH); Louisiana State University Museum of Natural Science, Baton Rouge, LA, USA (LSUMNS); National Museum of Natural History, Smithsonian Institution, Washington DC, USA (USNM); National Museum of Victoria, Australia (NMV); University of Kansas Museum of Natural History, Lawrence, Kansas, USA (KUMNH); University of Michigan Museum of Zoology, Ann Arbor, Michigan, USA (UMMZ); Museu de História Natural de Taubaté, Taubaté, São Paulo, Brasil (MHNT); e Museu Paraense Emílio Goeldi, Belém, Pará, Brasil (MPEG). O símbolo "?" representa os espécimes de sexo não identificado ou indeterminado.

Alcedinidae

Alcedininae

Alcedo atthis: AMNH 17749, fêmea; AMNH 22957, macho; MNHT 128, ?; UMMZ 119038, ?; UMMZ 119039, ?; USNM 343052, macho; USNM 562030, macho; USNM 605052, macho jovem; USNM 611824, fêmea; USNM 613064, fêmea.

Alcedo semitorquata: UMMZ 158188, fêmea.

Alcedo quadribrachys: AMNH 16332, ?; AMNH 18852, macho; USNM 291797, fêmea; USNM 322627, macho.

Alcedo meninting: AMNH 22958, ?.

Alcedo azurea: NMV B12713, macho; NMV B14217, fêmea; UMMZ 214243, macho; USNM 612709, macho; USNM 620236, macho.

Alcedo websteri: USNM 608680, macho; USNM 608681, fêmea; USNM 615064, macho.

Alcedo cyanopecta: UMMZ 204794, ?; UMMZ 204795, ?; USNM 613065, macho.

Alcedo cristata: AMNH 16898, macho; AMNH 16899, macho; UMMZ 220981, ?; UMMZ 220983, ?; USNM 347417, macho; USNM 491974, ?.

Alcedo leucogaster: AMNH 24709, fêmea; AMNH 24800, macho; USNM 292420, fêmea; USNM 318615, macho.

Ceyx lepidus: AMNH 6760, macho; AMNH 7397, ?; AMNH 7500, ?; USNM 557189, fêmea; USNM 615024, fêmea; USNM 615026, macho.

Ceyx erithacus: AMNH 22985, ?; USNM 321604, ?; USNM 560893, macho; USNM 559832, fêmea.

Ceyx rufidorsa: UKMNH 41129, fêmea; USNM 562034, fêmea; USNM 562035, ?.

Ceyx melanurus: UMMZ 204796, ?; USNM 562848, fêmea; USNM 613066, fêmea.

Ceyx fallax: USNM 226199, fêmea.

Ispidina picta: AMNH 5010, ?; UMMZ 223515, ?; UMMZ 223516, ?; USNM 291799, macho; USNM 430695, macho; USNM 488854, fêmea; USNM 491979, fêmea.

Ispidina lecontei: AMNH 16905, macho; AMNH 18849, fêmea; USNM 292421, macho; USNM 292422, macho.

Daceloninae

Dacelo novaeguineae: AMNH 9308, macho; AMNH 9408, fêmea; MHNT 126, ?; USNM 322826, macho; USNM 341814, macho; USNM 610568, macho; USNM 612710, fêmea; USNM 612711, fêmea; USNM 612712, fêmea.

Dacelo leachii: AMNH 4004, ?; AMNH 9094, fêmea; USNM 347672, fêmea.

Dacelo gaudichaud: AMNH 7383, fêmea; AMNH 7551, macho; UMMZ 215788, macho.

Pelargopsis capensis: UMMZ 204797, ?; USNM 320839, fêmea; USNM 343243, macho; USNM 562023, macho; USNM 562024, macho; USNM 562025, fêmea; USNM 562026, macho.

Pelargopsis melanorhyncha: AMNH 442, ?; USNM 226198, macho.

Halcyon coromanda: USNM 560894, macho; USNM 562027, macho; USNM 611825, fêmea; USNM 613067, macho.

Halcyon badia: AMNH 16907, macho; USNM 291323, macho; USNM 292418, macho.

Halcyon smyrnensis: AMNH 22986, ?; UMMZ 218527, ?; USNM 292070, fêmea; USNM 343265, macho; USNM 343266, macho; USNM 613068, macho; USNM 613071, fêmea.

Halcyon pileata: USNM 290198, fêmea; USNM 488645, macho.

Halcyon leucocephala (Müller, 1776): AMNH 4994, ?; USNM 322532, macho; USNM 322533, macho; USNM 431807, fêmea; USNM 488850, fêmea.

Halcyon senegalensis (Linnaeus, 1766): AMNH 24717, fêmea; AMNH 5839, ?; UMMZ 218550, ?; USNM 291098, macho; USNM 291099, fêmea; USNM 347423, macho; USNM 491981, fêmea.

Halcyon malimbica: AMNH 24801, fêmea; AMNH 16908, macho; USNM 292419, fêmea; USNM 318596, fêmea; USNM 347421, macho; USNM 347425, macho.

Halcyon albiventris (Scopoli, 1786): UMMZ 212930, ?; USNM 430688, fêmea; USNM 430802, macho; USNM 488847, fêmea. Halcyon chelicuti: UMMZ 208245, ?; USNM 322531, macho; USNM 431516, macho.

Todirhamphus nigrocyaneus (Wallace, 1862): USNM 614958, macho. 
Todirhamphus winchelli: UMMZ 207465, fêmea.

Todirhamphus diops (Temminck, 1824): USNM 557193, macho; USNM 557195, fêmea; USNM 558320, macho.

Todirhamphus macleayii (Jardine \& Selby, 1830): USNM 347661, macho; USNM 612713, macho; USNM 612714, fêmea.

Todirhamphus leucopygius (Verreaux, 1858): AMNH 23411, macho.

Todirhamphus pyrrhopygia (Gould, 1841): AMNH 9357, macho; AMNH 9336, macho; USNM 344792, macho; USNM 346624, fêmea; USNM 347659, fêmea.

Todirhamphus recurvirostris Lafresnaye, 1842. AMNH 8000, macho; AMNH 8001, macho.

Todirhamphus cinnamominus (Swainson, 1821): AMNH 22704, macho; USNM 431410, fêmea; USNM 612617, fêmea; USNM 615087, macho.

Todirhamphus funebris Bonaparte, 1850: USNM 557210, fêmea; USNM 557211, fêmea; USNM 560830, macho; USNM 560831, fêmea.

Todirhamphus chloris (Boddaert, 1783): AMNH 17750, fêmea; AMNH 25223, macho; UMMZ 204799, ?; USNM 290201, fêmea; USNM 560676, macho; USNM 562032, fêmea; USNM 562033, macho.

Todirhamphus sanctus (Vigors \& Horsfield, 1827): AMNH 6759, macho; UMMZ 214244, ?; USNM 557206, macho; USNM 557207, fêmea; USNM 561606, macho; USNM 620233, fêmea.

Todirhamphus ruficollaris (Holyoak, 1974): USNM 559590, macho; USNM 559591, fêmea.

Syma torotoro: LSUMNS 100988, ?.

Melidora macrorrhina: UMMZ 215786, ?.

Actenoides concretus (Temminck, 1825): USNM 431004, macho.

Actenoides lindsayi: UMMZ 204800, ?; USNM 613072, fêmea; USNM 613698, ?; USNM 613699, ?.

Tanysiptera galatea: USNM 557214, macho; USNM 557216, macho; USNM 557220, fêmea; USNM 557226, fêmea.

Tanysiptera sylvia: USNM 614960, fêmea.

Cerylinae

Megaceryle maxima: AMNH 8037, fêmea; AMNH 16331, macho; USNM 560529, fêmea.

Megaceryle lugubris: USNM 292776, macho; USNM 318238, ?; USNM 319068, macho.

Megaceryle alcyon: AMNH 18286, fêmea; AMNH 24596, macho; UMMZ 107228, ?; UMMZ 107339, ?; USNM 322728, fêmea; USNM 553851, macho; USNM 610992, jovem, sexo indeterminado; USNM 610994, macho; USNM 610995, macho jovem; USNM 621370, fêmea.

Megaceryle torquata: AMNH 447, ?; AMNH 4654, ?; MHNT 127, fêmea; MHNT 378, fêmea; MHNT 1500, fêmea; USNM 288829, fêmea; USNM 345913, macho; USNM 345914, fêmea; USNM 500429, macho; USNM 562559, macho.

Ceryle rudis: AMNH 4228, ?; UMMZ 204267, ?; UMMZ 206585, ?; USNM 322468, macho; USNM 322469, fêmea; USNM 322470, macho; USNM 430632, macho; USNM 430686, fêmea.

Chloroceryle amazona: AMNH 13816, macho; MHNT 306, fêmea; USNM 227695, fêmea; USNM 559478, macho; USNM 562204 , macho; USNM 613998, fêmea; USNM 613999, fêmea.

Chloroceryle americana: AMNH 8556, macho; MHNT 611, fêmea; MHNT 628, macho; MHNT 767, macho; USNM 500476, macho; USNM 560164, macho; USNM 562562, fêmea; USNM 614650, fêmea.

Chloroceryle inda: MHNT 1371, macho; FMNH 315406, macho; FMNH 318863, macho; LSUMNS 118212, ?.; UMMZ 154727, ?; UMMZ 154728, ?; USNM 562208, fêmea; USNM 613418, fêmea; USNM 621406, macho; USNM 622337, macho.

Chloroceryle aenea: AMNH 8324, macho; AMNH 8326, fêmea; MHNT 609, macho; MHNT 1416, fêmea; USNM 492397, macho; USNM 500553, macho; USNM 502492, fêmea; USNM 562563, fêmea; USNM 614001, macho.

Meropidae

Nyctyornis amictus: USNM 18766, ?; USNM 18767, ?

Nyctyornis athertoni: LSUMNS 118818, ?; USNM 343253, macho.

Meropogon forsteni: USNM 226194, fêmea.

Merops gularis: USNM 291107, fêmea; USNM 291108, fêmea.

Merops muelleri: USNM 431818, fêmea.

Merops bulocki: AMNH 4222, ?; AMNH 4223, ?; USNM 322418, fêmea; USNM 322420, macho.

Merops bullockoides: USNM 430452, macho.

Merops pusillus: AMNH 24793, fêmea; USNM 430366, macho; USNM 501336, ?.

Merops variegatus: AMNH 24794, macho; USNM 322421, fêmea; USNM 561963, macho.

Merops oreobates: USNM 431678, fêmea; USNM 431816, macho.

Merops hirundinaceus: UMMZ 219890,?.

Revista Brasileira de Zoologia 23 (3): 841-864, setembro 2006 
Merops albicollis: USNM 347438, ?; USNM 491983, macho.

Merops orientalis: USNM 292225, fêmea; USNM 343248, fêmea; USNM 343252, macho.

Merops persicus Pallas, 1773: USNM 347436, macho.

Merops superciliosus: MHNT 1452, ?; USNM 343523, fêmea.

Merops philippinus: USNM 225823, macho; USNM 613073, macho.

Merops ornatus: AMNH 7601, fêmea; AMNH 9649, macho; USNM 557238, fêmea; USNM 620239, ?.

Merops apiaster: AMNH 4209, ?; AMNH 23335, fêmea; MHNT 131, macho; MHNT 1356, ?; USNM 552948, macho; USNM 603624, fêmea; USNM 603625, fêmea.

Merops malimbicus: FMNH 313085, macho; FMNH 313292, fêmea.

Merops nubicus: AMNH 4233, ?; USNM 322454, fêmea; USNM 621686, macho.

Todidae

Todus multicolor: USNM 562474, macho; USNM 562475, macho; USNM 612249, macho.

Todus angustirostris: AMNH 25420, fêmea; AMNH 25421, macho; USNM 553413, fêmea; USNM 554620, macho; USNM 555793, macho; USNM 555794, fêmea.

Todus mexicanus: USNM 501846, macho; USNM 501847, fêmea; USNM 501848, macho; USNM 553304, fêmea.

Todus todus: USNM 502755, fêmea; USNM 558883, macho; USNM 558886, macho; USNM 559190, fêmea.

Todus subulatus Gray, 1847. AMNH 25466, macho; AMNH 25467, fêmea; MHNT 129, ?; USNM 554624, macho; USNM 554625, macho; USNM 555798, fêmea; USNM 555801, macho.

Momotidae

Momotus momota: USNM 428842, macho.

Baryphthengus ruficapillus (Vieillot, 1818): USNM 429819, macho; USNM 613420, fêmea.

Baryphthengus martii (Spix, 1824): LSUMNS 89851, fêmea; LSUMNS 111178, macho; USNM 614002, macho.

Aspatha gularis: UMMZ 151586, macho; USNM 343481, macho.

Hylomanes momotula: LSUMNS 104620, macho; LSUMNS 108753, fêmea.

Eumomota superciliosa: KUMNH 37616, macho.

Electron platyrhynchum: USNM 321526, ?; USNM 428583, macho.

Electron carinatum: KUMNH 34812.

Coraciidae

Coracias garrulus: AMNH 457, ?; AMNH 12839, macho jovem; USNM 552952, fêmea; USNM 552953, fêmea; USNM 552954, macho; USNM 552955, macho.

Coracias abyssinica Hermann, 1783: FMNH 320012, macho; FMNH 320014, macho.

Coracias caudata: AMNH 1471, ?; AMNH 3759, macho; MHNT 134, ?; MHNT 1612, macho; USNM 431907, macho; USNM 431908, fêmea; USNM 489393, macho; USNM 621688, fêmea.

Coracias spatulata: UMMZ 214366, ?.

Coracias noevia: AMNH 1469, ?; LSUMNS 28039, macho.

Coracias benghalensis (Linnaeus, 1758): AMNH 8223, ?; AMNH 8561, ?; USNM 343054, macho; USNM 343284, fêmea; USNM 490190, fêmea.

Coracias temminckii (Vieillot, 1819): USNM 226015, macho.

Coracias cyanogaster Cuvier, 1817: FMNH 320018, fêmea; FMNH 320020, macho.

Eurystomus glaucurus (Müller, 1776): AMNH 24802, fêmea; USNM 223862, ?; USNM 347416, fêmea.

Eurystomus gularis: USNM 292413, macho; USNM 292414, fêmea.

Eurystomus orientalis (Linnaeus, 1766): AMNH 7544, macho; AMNH 7628, macho; MHNT 133, fêmea; USNM 432045, macho; USNM 488903, fêmea; USNM 560141, ?; USNM 562037, fêmea; USNM 620238, fêmea.

Eurystomus azureus: USNM 560832, macho jovem; USNM 560834, macho; USNM 560835, fêmea.

Brachypteraciidae

Brachypteracias leptosomus: USNM 223863, ?.

Atelornis pittoides: UMMZ 208409, macho.

Atelornis crossleyi: USNM 223864, macho.

Leptosomidae

Leptosomus discolor: AMNH 448, ?; USNM 291844; USNM 291845, fêmea.

Revista Brasileira de Zoologia 23 (3): 841-864, setembro 2006 
Phoeniculidae

Phoeniculus purpureus: AMNH 4229, ?; USNM 320948, fêmea; USNM 558553, ?; USNM 559060, fêmea.

Phoeniculus bollei: AMNH 4232, ?; KUMNH 70844, fêmea; LSUMNS 28041, macho; LSUMNS 28042, macho.

Rhinopomastus cyanomelas: UMMZ 156677, ?; UMMZ 214365, macho; USNM 431714, macho; USNM 431715, fêmea.

Rhinopomastus minor: KUMNH 70670, fêmea; UMMZ 156075, ?; UMMZ 156678, fêmea; USNM 431721, fêmea.

Upupidae

Upupa epops: AMNH 8768, ?; MHNT 135, ?; MHNT 399, ?; USNM 289547, macho; USNM 290310, macho; USNM 292064, macho; USNM 292930, fêmea; USNM 343282, macho; USNM 344367, fêmea; USNM 603627, macho; USNM 603629, fêmea; USNM 603632, fêmea; USNM 603634, fêmea; USNM 603636, macho; USNM 603637, macho; USNM 603638, fêmea; USNM 603650, fêmea; USNM 603657, fêmea; USNM 603666, macho.

Bucerotidae

Tockus hartlaubi: USNM 322438, ?.

Tockus bradfieldi (Roberts, 1930): USNM 432026, macho.

Tockus camurus: USNM 291103, fêmea.

Tockus erythrorhynchus: USNM 291161, macho; USNM 343133, fêmea.

Tockus [flavirostris] leucomelas (Lichtenstein, 1842): USNM 558556, macho; USNM 558557, fêmea.

Tockus deckeni: USNM 431796, fêmea; USNM 431798, macho.

Tockus fasciatus: USNM 291102, fêmea.

Tockus albocristatus (Cassin, 1848): USNM 291104, fêmea; USNM 322630, macho.

Tockus nasutus (Linnaeus, 1766): USNM 430786, macho; USNM 490237, fêmea.

Tockus alboterminatus (Büttikofer, 1889): USNM 290510, macho; USNM 429190, macho.

Ocyceros [griseus] griseus (Latham, 1790): USNM 347601, macho.

Ocyceros birostris (Scopoli, 1786): USNM 347342, macho.

Anthracoceros malayanus (Raffles, 1822): USNM 19927, macho.

Anthracoceros marckei Oustalet, 1885: USNM 562039, macho; USNM 562040, fêmea.

Anthracoceros coronatus (Boddaert, 1783): USNM 19475, macho; USNM 344622, fêmea; USNM 430177, fêmea.

Anthracoceros malabaricus (Gmelin, 1788): USNM 292223, macho; USNM 343270, fêmea.

Buceros rhinoceros: USNM 345082, macho; USNM 346727, fêmea.

Buceros bicornis: USNM 19465, ?, jovem; USNM 19619, macho; USNM 432505, fêmea; USNM 430617, fêmea.

Buceros hydrocorax: USNM 223896, ?; USNM 556260, macho; USNM 613076, fêmea.

Buceros vigil: USNM 18756, ?; USNM 19886, macho.

Anorrhinus galeritus: USNM 18758, ?; USNM 19479, ?; USNM 343179, macho.

Penelopides panini (Boddaert, 1783): USNM 291383, fêmea; USNM 613074, ?; USNM 613075, macho.

Penelopides exarhatus (Temminck, 1823): USNM 226017, macho.

Aceros comatus: USNM 344798, macho.

Aceros [leucocephalus] leucocephalus (Vieillot, 1816): USNM 430273, fêmea; USNM 488897, macho; USNM 488898, macho.

Aceros [leucocephalus] corrugatus (Temminck, 1832): UMMZ 219873, fêmea.

Aceros cassidix: USNM 224805, macho.

Aceros undulatus (Shaw, 1811): USNM 19687, macho; USNM 344519, fêmea.

Aceros plicatus: USNM 320833, fêmea; USNM 558296, macho.

Ceratogymna fistulator (Cassin, 1852): USNM 347441, fêmea.

Ceratogymna subcylindricus (Sclater, 1870): USNM 227121, macho.

Ceratogymna bucinator (Temminck, 1824): USNM 322587, macho; USNM 490179, fêmea.

Ceratogymna cylindricus (Temminck, 1824): USNM 291101, macho; USNM 291325, fêmea.

Ceratogymna atrata (Temminck, 1835): USNM 224821, fêmea; USNM 291326, fêmea; USNM 431061, macho.

Ceratogymna elata: USNM 4710, macho; USNM 345443, jovem, sexo indeterminado.

Ceratogymna brevis: USNM 430540, macho; USNM 614885, ?

Bucorvus abyssinicus: USNM 321839, macho; USNM 346438, fêmea; USNM 346457, fêmea.

Bucorvus cafer: USNM 429091,?.

Recebido em 31.I.2006; aceito em 30.VIII.2006.

Revista Brasileira de Zoologia 23 (3): 841-864, setembro 2006 